\title{
Long-term persistence of crAss-like phage crAss001 is associated with phase variation in Bacteroides intestinalis
}

\section{Short title: Mechanisms of persistence of a crAss-like phage}

Andrey N. Shkoporov*1, Ekaterina V. Khokhlova*1, Niamh Stephens ${ }^{2}$, Cara Hueston ${ }^{1}$, Samuel Seymour ${ }^{1}$, Andrew J. Hryckowian ${ }^{3}$, Dimitri Scholz ${ }^{2}$, R. Paul Ross ${ }^{1}$, Colin Hill ${ }^{1}$

*These authors contributed equally to the study.

10 1. APC Microbiome Ireland and School of Microbiology, University College Cork, Cork, Ireland

2. Conway Institute of Biomolecular and Biomedical Research, University College Dublin, Belfield, Dublin 4, Ireland

3. Division of Gastroenterology and Hepatology, Department of Medicine; Department of Medical Microbiology \& Immunology, University of Wisconsin School of Medicine and Public Health,

15 Madison, WI, USA

\section{Abstract}

The crAss-like phages are ubiquitous and highly abundant members of the human gut virome that infect commensal bacteria of the order Bacteroidales. Although incapable of classical lysogeny,

20 these viruses demonstrate unexplained long-term persistence in the human gut microbiome, dominating the virome in some individuals. Here we demonstrate that rapid phase variation of alternate capsular polysaccharides plays an important role in dynamic equilibrium between phage sensitivity and resistance in $B$. intestinalis cultures, allowing phage and bacteria to multiply in parallel. The data also suggests the role of concomitant phage persistence mechanisms associated with delayed lysis of infected cells, such as carrier state infection. From an ecological and evolutionary standpoint this type of phage-host interaction is consistent with the Piggyback-theWinner model, which suggests a preference towards lysogenic or other "benign" forms of phage infection when the host is stably present at high abundance.

30 Keywords: crAss-like phages; human gut microbiome; human virome; phase variation; capsular polysaccharides; carrier state infection.

Teaser: CrAss-like phage persistence in Bacteroides is associated with capsule phase-variation and additional unexplored mechanisms.

\section{Introduction}

The crAss-like bacteriophages are a recently described family of dsDNA tailed bacterial viruses of the order Caudovirales that are predicted to infect bacteria of the phylum Bacteroidetes (1). They are present in variety of host-associated, aquatic and terrestrial habitats, but are especially predominant in the human gut (2-4). The presence of the prototypical crAssphage (p-crAssphage) in $>70 \%$ of human gut metagenomes together with its exceptional abundance in some of the samples ( $>90 \%$ of the gut virome and $>20 \%$ of total faecal DNA) led to its description as "the most abundant virus of the human body" (4). Furthermore, p-crAssphage was found to be widespread in the global human population with sequence variants correlating with geographic location (5). Based 
45 on the observation of related genomes in the non-human primate gut, it was proposed that pcrAssphage shares a long co-evolutionary history with the human species. In addition, limited dietary associations, but no link to disease was observed in crAssphage colonisation patterns (3). A recent longitudinal study of the human gut virome highlighted long-term (months and even years) high-level persistence of different combinations of crAss-like phages across individuals (6). When

50 vertically transmitted from mothers to their infants or transplanted into in the course of faecal microbial transplantation (FMT), crAss-like phage engraft successfully and stably persist for many months $(7,8)$. CrAss-like phages can also persist in pure bacterial culture in vitro over many serial passages without any significant effect on the density of host population (9). The overall effect of stably persisting crAss-like phages, sometimes reaching absolute concentrations of $10^{11}$ genome

55 copies per gram of faeces (6), on the structure (composition) and function of the gut microbiome is yet to be determined. Despite a lack of disease associations with p-crAssphage, correlations with other candidate genera and individual strains within the family of crAss-like phages has not been examined. Certain recent studies hinted at depletion of certain crAss-like viral clusters in an IBD patient cohort (10), while at the same time a genus VI crAss-like phage, termed IAS virus, was

60 prevalent in HIV patients with unexplained diarrhoea (11).

It remains unclear what mechanisms are responsible for the long-term persistence and high prevalence of crAss-like phages in the human gut. Several mechanisms of phage persistence are possible, including spatial heterogeneity of microbial habitats in the gut (12), physiological stochasticity of phage sensitivity phenotype in their host bacteria (13), reversible genetic switching

65 (phase variation) of surface receptor expression (14), constant sweeps of new mutations leading to arms race co-evolution with their hosts (15), or perhaps, an unusual life cycle (carrier state infection, pseudolysogeny) of the viruses themselves.

Establishing these mechanism(s) is a challenging task due to the lack of tools for genetic manipulation, and the low similarity of crAss-like phage genomes and proteins to well characterised

70 model viruses (16). Our limited understanding of biological properties of crAss-like phages derives from crAss001, DAC15, and DAC17, the first three cultured human crAss-like phages (all members of the candidate genus VI) $(9,17)$.

Here, we report the first comprehensive effort to characterise interaction of crAss-like phages with their hosts, focusing primarily on crAss001. We demonstrate that phase variation of capsular

75 polysaccharides creates dynamic sub-populations of sensitive and resistant Bacteroides cells, driving long term phage persistence. A yet unexplained mechanism is apparently responsible for a paused infection cycle and delayed release of phage progeny from a significant fraction of infected cells, a phenomenon that may represent a novel type of carrier state infection.

\section{Results}

\section{CrAss-like phages are capable of long-term stable persistence in the mammalian gut and select for resistance in their bacterial host}

We have previously demonstrated the long-term persistence of crAss-like phages in the human gut microbiome (6). The levels of crAss-like phage colonisation in ten subjects varied from $<10 \%$ of the total faecal virome to nearly $100 \%$. Eight of the ten subjects were stably colonised by one or several individual crAss-like phage strains.

Different crAss-like phage genera tended to colonise with distinctive relative abundances. High level colonisation with relative abundances approaching $100 \%$ of the virome were typical of genus 
I, whereas genus VI had much lower relative abundance, possibly reflecting differences in their host abundance, mode of replication, or the rate of emergence of resistance in the host strains (Fig. 1a). To reinforce this observation we followed the dynamics of crAss-like phage persistence in a monoxenic mouse model using a cultivable representative of the family, $\Phi$ crAss001. Mice $(n=6)$ colonised with $B$. intestinalis alone served as a negative control. Within the first few days upon bacterial gavage, faecal $B$. intestinalis reached levels of $10^{11} \mathrm{cfu} \mathrm{g}^{-1}$ regardless of the presence of phage ( $>0.05$ in Kruskal-Wallis test with FDR correction for all time points except day 31). ФcrAss001 propagated to much lower levels of $10^{6}-10^{8} \mathrm{pfu} \mathrm{g}^{-1}$. However, this level also remained until the termination of the experiment at 136 days (Fig. 1b).

Long term persistence of the phage-host pair quickly induces resistance to $\Phi$ crAss001 in $B$. intestinalis. By day three from the first gavage, 11/17 (65\%) of B. intestinalis faecal isolates were

100 completely resistant to phage in spot and plaque assays, compared to $2 \%$ of spontaneously resistant cells in an overnight broth culture grown in the absence of phage (see below). By week twelve, 15/29 (52\%) isolates were resistant. The sensitive isolates showed varied degrees of partial resistance that was manifested by formation of spots of variable turbidity. Unexpectedly, even bacterial isolates from the control animals showed a switch towards the phage-resistant phenotype

105 in that while day three isolates were all sensitive (17/17), 8/29 (27\%) isolates from week twelve were completely resistant, showing that a switch between sensitive and resistant phenotypes can occur in the host strain independently of phage attack.

This persistence can be recapitulated in vitro, in a B. intestinalis culture infected in its early logarithmic phase at multiplicity of infection (MOI) of 1, followed by overnight incubation and

110 daily passages of resulting phage-bacterial co-culture. Initial absorption of phage resulted in $96.5 \%$ reduction of bacterial CFU after $30 \mathrm{~min}$ (Fig. 1c), followed by the first round of phage genome replication, which was largely completed by $90 \mathrm{~min}$ after infection resulting in a calculated virus-tomicrobe ratio (VMR) of $\sim 22.8 \pm 1.2$ (median \pm IQR, Fig. 1d). Parallel propagation of bacteria and phage for the first 24 hours maintained roughly the same level of VMR and resulted in high titre of

115 phage particles $3.86 \times 10^{10} \pm 7 \times 10^{9} \mathrm{pfu} \mathrm{ml}^{-1}$ (Fig. 1bc). Subsequent daily transfers led to a drop of VMR and phage titres, but resulted in increased viable bacterial titres and a phage/host dynamic that is stably maintained for as many as 23 daily transfers (Fig. 1d). Agreeing with previous observations in non-crAss-like phages and Bacteroides thetaiotaomicron (14), this evidence again points towards a rapid shift to dominance by resistant cells with retention of a sufficient sensitive sub-population to allow for low level phage reproduction and persistence.

\section{Resistance of B. intestinalis cells to $\Phi$ crAss001 develops at high rate in vitro, but is reversible and associated with inhibition of phage adsorption}

ФcrAss001 forms turbid spots in soft agar overlays containing B. intestinalis APC919/174,

125 confirming that bacterial growth can occur in the presence of phage (Fig. 2a). This could be a result of a high rate of lysogeny, or of a pre-existing resistant sub-population. resistant sub-population. To distinguish between these possibilities, transmission electron microscopy (TEM) was conducted on material collected from the center of the spot shown in Fig. 2a. Consistent with the hypothesis that a pre-existing resistant subpopulation of bacteria are present in the spot material, the electron

130 micrographs revealed the presence of both uninfected and infected cells among the cell debris, including many instances in which cells in the process of division had phage progeny restricted to only one of the two nascent daughter cells (Fig. 2b-f).

In the presence of an excess of phage in soft agar overlays (efficiency of plating assay, EOP), clonal cultures of $B$. intestinalis APC919/174 had an immediate resistance rate of $2.06 \pm 0.14 \%$ (mean \pm SD, 
135 Fig. 2g-h), which agrees with 96.5\% cells being infected above. None of the tested colonies contained DNA of $\Phi$ crAss001 detectable by PCR. When ten resistant clones were each sub-cultured into 12 separate clones, four of the 120 (3.3\%) reverted to sensitive phenotype. Resistant clones demonstrated a dramatically reduced ability to adsorb phage, while the spontaneously sensitive 'revertants' had restored phage adsorption (Fig. 2j). Some of the sub-clones had altered colony

140 morphologies, with resistant colonies being more transparent, while sensitive ones were opaque (e.g. sub-clones $8 \mathrm{~T}$ and $8 \mathrm{~W}$ in Fig. 2i). When a resistant clone $8 \mathrm{~T}$ was subjected to an EOP assay the rate of survival was raised to $88.7 \pm 3.3 \%$, but in the sensitive sub-clone $8 \mathrm{~W}$ it had returned closer to the original level of $11.3 \pm 0.9 \%$. TEM of $8 \mathrm{~T}$ cells revealed an altered "hairy" appearance, with a loose and bulky polymeric substance indicative of production of a different type of capsular

145 polysaccharide (CPS) (Fig. 2k). In contrast, the wild-type (WT) culture demonstrates a much more compact thinner layer of capsular polysaccharide intimately attached to the outer membrane (Fig. 2l).

Together, these data suggests that cultures of $B$. intestinalis contain mixed populations of cells. The transient phage-resistant phenotype results from constantly ongoing reversible switching of

150 expression of cell surface structures through some genetic or epigenetic mechanism. Approximately $2 \%$ of cells in naïve cultures were resistant to phage. Exposure to phage in continuous culture in vitro or in the murine gut drives the bacterial population towards phage resistance, but this resistance does not reach $100 \%$ or lead to the extinction of the phage under the conditions tested.

155 Phase variation of multiple surface loci and concomitant capsular polysaccharide switching in

\section{B. intestinalis}

In order to identify the mechanism of resistance we subjected ten resistant clones and two revertant sensitive sub-clones to whole genome sequencing. We also performed sequencing of samples collected from the long-term in vitro persistence experiment $5 \mathrm{~min}-28$ days following the

160 inoculation and some of the faecal samples collected in long term murine colonisation experiment. For the wild type strain, resistant clone 8T ("transparent") and its corresponding revertant clone 8W ("white"), complete circular genomes were assembled. A comparison of the three assemblies revealed that the only detectable differences consist of the inversion and rearrangement of several genomic loci (Fig. S1a). Similar to previous observations with B. thetaiotaomicron (14), three types

165 of events were detectable, including, (a), large inversions involving entire gene clusters comprised of genes coding for TonB-dependent transporters (18) and/or RagB/SusD-like nutrient uptake proteins (19), often flanked with a dedicated integrase/recombinase gene; (b), a more complex type of reshuffling of clusters containing ABC-transporter genes (Fig. S1b); and (c), the inversion of small, <200 bp regions adjacent to capsular polysaccharide biosynthesis operons that contain

170 potential promoters. Remarkably, all these genomic loci, designated as "phase variable regions" (PVRs), can be directly implicated in phage sensitivity. Their encoded products are either components of cell surface structures with potential to serve as receptors or barriers for phage adsorption or potential phage defence molecules (e.g. restriction/modification system). Transcriptomic analysis of serial daily broth co-cultures of $B$. intestinalis and ФcrAss001 1-5 days

175 after the initial inoculation revealed an array of genes showing divergent responses to the presence of phage $(\mathrm{p}<0.01, \mathrm{DESeq} 2)$. The strongest transcriptional response in the host cells, already visible after the first $24 \mathrm{~h}$, was associated with PVRs and consisted in the strong 100-fold repression of the PVR9 capsular polysaccharide (CPS) biosynthesis operon, the up-regulation of similar CPS operons associated with loci PVR7, PVR8 and PVR11 (Fig. 3a), and the up-regulation of a group of 180 nutrient uptake genes in a complete inversion of the integrase/recombinase gene-flanked operon 
PVR3. Importantly, similar alterations of gene expression patterns relative to the phage-naïve WT culture of $B$. intestinalis can be observed in the resistant clone 8T (in the absence of phage). Its phage sensitive derivative 8W displays restored levels of PVR9 expression, down-regulated (compared to WT) PVR7, PVR8 and PVR11 and up-regulated expression of PVR3.

185 The CPS-encoding loci PVR7-11 have broadly differing gene content with limited levels of amino acid homology between certain shared protein orthologues (e.g. oligosaccharide flippases, dehydrogenases, dehydratases and glycosyl transferases; Fig. 3b). All of these loci share a putative invertible, moderately conserved promoter sequence of 183-186 bp (Fig. 3c) flanked by a perfect, completely conserved 10 bp inverted repeat GTTCGTTTAA at which recombination most likely occurs. This sequence partially overlaps with inverted repeats ARACGTTCGTN flanking phase variable promoters controlled by a serine recombinase family, master DNA invertase (mpi) in Bacteroides fragilis (20). At least two homologs of this enzyme are encoded in the genome of $B$. intestinalis APC919/174 (NCBI accession QDO70032.1 and QDO69715.1). A search for similar patterns in the B. intestinalis APC919/174 genome revealed the presence of an additional, fifth, CPS 195 locus (denoted PVR12) with identical inverted repeat flanking its promoter region. This locus, however, did not show significant up- or down-regulation of expression in our RNAseq assays. By mapping the RNAseq reads we located the approximate transcription start in PVR7-11 inside the proximal copy of the inverted repeat (Fig. 3c), confirming that the invertible regions are capable of acting as promoters.

200 To explore the rate and frequency of phase variation on the complete genome scale and to identify other potential PVRs, we performed local alignment of individual long DNA reads (obtained from WT culture, resistant clones 8T and PhR5, and corresponding revertant sub-clones 8W and PhR5-1) to the assembled circular genome reference, searching for cases where parts of the same long read align inconsistently, indicating either inversion or translocation. This analysis revealed a series of

205 strong recombination hotspots present in every culture tested. The majority were associated with already known PVRs, with exception of a few novel finds, one of which is the previously mentioned fifth CPS locus PVR12 (Fig. S2). We then used mapping of short shotgun reads to allow the quantitation of inversions in the long term in vitro and in vivo persistence experiments, ten resistant clones and one revertant sub-clone. By aligning paired-end short reads we calculated the

210 relative proportions of cells with two opposite orientations of the invertible promoters of PVR7-12 in different cultures of B. intestinalis APC919/174 (Fig. 3d-e). We found that the sensitive WT culture is characterised by PVR9 promoter mainly in the ON orientation, while PVR7, PVR8, PVR11 and PVR12 are mainly OFF. In agreement with the RNAseq data, the resistant clone 8T shows a switch of CPS expression from PVR9 towards PVR7, while the WT genotype is largely

215 restored in sensitive revertant sub-clone 8W. Interestingly, other resistant clones showed the PVR9 promoter in mostly the ON orientation, combined with an ON state in one of the three other CPSassociated PVRs. Variable patterns of promoter activation with PVR9 being ON were detectable in the long term in vitro and in vivo persistence experiments (Fig. 3d, Fig. S3).

We suggest that in the $B$. intestinalis- $\Phi$ crAss001 phage-host pair some of the CPS can be

220 permissive to phage infection, and even serve as phage receptors (possibly PVR9), while some other can be protective (PVR7, PVR8, PVR11 and PVR12) or neutral. This is similar to what was observed for a large panel of non-crAss-like phages (14), as well as crAss-like-phages DAC15 and DAC17 in B. thetaiotaomicron (17). We conclude that phase variation of CPS expression leading to phage resistance occurs spontaneously, even in the absence of phage. As a result, a dynamic 225 equilibrium between sensitive and resistant sub-populations is maintained, which can be shifted 
towards the resistance phenotype by phage-driven selection. However, a constant switching back to sensitivity provides the phage with a constant supply of sensitive cells, thus assuring its long-term persistence.

\section{Delayed release of ФcrAss001 progeny leads to multiple echo phage bursts and potentially provides an infection escape mechanism}

We previously reported a remarkably low apparent burst size of only 2.5 pfu per cell infected with ФcrAss001 (9). This cannot be explained by failure of phage to infect the majority of cells as only $\sim 2 \%$ cells in naïve $B$. intestinalis cultures were phage-resistant due to phase variation. At the same

235 time, >50 particles per cell are visible in TEM images from ultrathin $(80 \mathrm{~nm})$ cell sections (Fig. 2b, d, f) and at least 20 new copies of phage genome are produced in an infected culture per copy of bacterial genome after 90 min of infection (as shown by metagenomic sequencing, Fig. 1d), ruling out the possibility of extremely low progeny counts per infected cell. Further to that, the efficiency of centre of infection tests (EOCI), showed that $56.6 \pm 12.7 \%$ of cells infected at an MOI=1, and

$24059.16 \pm 27.9 \%$ infected at an $\mathrm{MOI}=10$, were capable to give rise to infection centres (plaques) in $B$. intestinalis lawns. Electron microscopic observations of cultures infected at an $\mathrm{MOI}=1$ reveal that $>90 \%$ of cells show signs of early stages of virion assembly process 40 and 90 minutes after infection (Fig. S4). Therefore, the observed low burst size cannot be explained by phase variationbased resistance or low progeny per cell counts and must from an unknown mechanism limiting

245 replication and/or release of phage progeny from a significant fraction of infected cells. In order to get insights into this mechanism, we performed strand-specific RNAseq analysis of a single phage replication cycle in $B$. intestinalis cells infected at an MOI of 1 . Three transcriptional modules can be observed in the $\Phi$ crAss001 genome, which also correlate with ORF orientation and the putative operon organisation of the genome (Fig. 4a). The expression of the early genes, located on the right end of the linear phage genome, is initiated between 0 and 10 minutes after phage infection. The predicted products of these genes include a transcriptional regulator, two conserved domain proteins with unknown function and a TROVE domain protein (possible ribonucleoprotein component). A recent study in a distantly related marine crAss-like phage $\Phi 14: 2$ infecting Cellulophaga baltica indicated that a similarly organised operon of early genes is transcribed by a giant virion-associated multi-subunit RNA polymerase [RNAP, (21)], ejected into the cell in the course of infection (22). A homologue of this giant polymerase is present in all crAss-like phages and is also encoded by three large ORFs located in the central part of ФcrAss001 genome (4, 9, 23). Proteomic analysis of the crAss001 virion also indicated the presence of the subunits of the giant polymerase in the assembled phage particle (9).

260 Transcription of the middle and late genes is initiated between 10-30 and 30-60 minutes, respectively, and is likely to depend on the action of the host RNAP [as suggested for $\Phi 14: 2$ in (21)]. The middle genes are largely composed of functions associated with DNA replication and recombination, as well as nucleotide metabolism. Other genes of the same class can be involved in the degradation of host DNA and mRNA (HKD family nuclease, HicA mRNAse). The late genes, occupying the left-hand half of the genome, mainly encode structural proteins of the virion head and tail, as well as proteins participating in the virion assembly (terminase, chaperone) and cell lysis (holin, endolysin). Large ORFs coding for virion-associated RNAP subunits are located in the approximate centre of the linear genome and are transcribed during middle (gp49, predicted catalytic subunit, and gp50) or late (gp47) transcriptional stages. It is unclear why the temporal 
270 separation in RNAP subunit production might be needed. It may be that gp49 and gp50 are involved in transcription of the late genes in the same infection cycle in which they are produced, whereas gp47 is needed solely for the early genes transcription in the next cycle, or plays a role in the packaging, ejection or activation of the RNAP holoenzyme.

Interestingly, there was significant overlap in transcription of early, middle and late genes: once

275 activated, transcripts of each of the three modules persisted through the rest of the replication cycle

(Fig. 4a-b). The host transcriptional response was largely confined to strong genome-wide inhibition of transcription, occurring between 10-30 minutes preceded by the activation of certain energy metabolism functions at 10 minutes (most notably citrate synthase and NADP-dependent iso-citrate dehydrogenase, Fig. $\mathbf{4 b}$ ).

280 Morphological changes in the infected cells were visible as early as after 45 minutes as the formation of variable-sized electron-light spots (Fig. 4c). Ninety minutes into the infection cycle, well after the onset of expression of structural genes, the first intact phage particles (electron-dense spots) begin to appear amongst the electron-light spots. Large clusters of viral particles are visible inside many of the infected cells at 120 minutes. Agreeing with the one step growth curves (Fig. 4d-

285 e), cell lysis becomes noticeable at 150 minutes and widespread at 180 minutes. Interestingly, however, a large fraction of cells at 150 minutes remain intact, and only show signs of the early stage of infection with no properly formed phages particles (Fig. 4f). This observation can be interpreted as de-synchronisation of the phage replication cycle with some of the cells falling behind the others in terms of infection stage. Supporting this hypothesis is the continued expression 290 of early and middle operon genes, overlapping in timing with late genes. This is further reflected in the one step growth curves, where at an MOI of 1 or 10, a series of discrete, evenly spaced bursts are apparent with a low total progeny output ( 3-6 pfu per capita).

Recently, bacteriophages similar to $\Phi$ crAss001 in morphology and genome organisation (Fig. S5ab) were isolated against $B$. thetaiotaomicron (17). Phages DAC15 and DAC17 are also capable of

295 long-term persistence in serial broth cultures of B. thetaiotaomicron VPI-5482 (Fig. S5c) and do not significantly reduce the bacterial population. While the wild-type strain produce eight different phase-variable CPS, a panel of engineered mutants have been developed, which included an acapsular version, as well as eight strains expressing only one of the eight different CPSs, referred to hereafter as CPS1-8 $(24,25)$. Phages DAC15 and DAC17 were reported to infect the $c p s 3^{+}$

300 mutant efficiently, while only showing weak replication in WT VPI-5482 and other single-CPS expressing derivatives, indicating that CPS3 plays a permissive role in phage infection. In our hands, both DAC15 and DAC17 were able to persist stably in serial daily broth cultures of cps3 $^{+}$, as well as in WT (albeit at lower levels, Fig. S5d). This is despite the fact that an inability to switch to expression of alternative CPSs leads to a very low fraction of pre-existing resistant cells

$305\left(<10^{-5}\right)$ in populations of $c p s 3^{+}$, compared to $52.8 \pm 1.2-64.2 \pm 3.0 \%$ of cells resistant to DAC15/ DAC17 in the WT strain. This indicates that the removal of CPS variability leads to greatly reduced resistance to phage but does not eliminate the ability of phage to persist long-term. In one step growth experiments at an MOI=1, DAC15 and DAC17 behaved very similarly to ФcrAss001, producing a series of bursts and a low apparent output of progeny (Fig. S5e-f). However, unlike in

310 B. intestinalis- $\Phi$ crAss001 system, infection of $c p s 3^{+}$mutant at MOI=10 resulted in a single burst of $\sim 160$ pfu per capita, a value which is in a much closer agreement with the progeny size observed microscopically (Fig. S5g). 
These data suggests that a mechanism of gradual release of phage progeny operates in Bacteroides cells infected with crAss-like phages ФcrAss001, DAC15 and DAC17. This mechanism is largely independent of the dynamic phase variation mediated resistance discussed above. It appears that at high MOI the mechanism might become overwhelmed, or that the stress response in cells simultaneously infected by multiple phage particles switches it off. This leads to abrupt cell lysis and release of large phage progeny simultaneously, in a "classical” virulent phage manner.

\section{Discussion}

Since their discovery in 2014, crAss-like phages have become one of the most intriguing elements of the human virome. Based largely on metagenomic observations, this group of tailed dsDNA bacteriophages differs significantly from other members of the order Caudovirales in terms of their genome organisation, gene content, virion morphology, life cycle and ecological distribution (3, 6,

325 9, 16). Of special interest is their unexplained, overwhelming prevalence in the gut microbial community, their ability for long term persistence and seemingly benign interaction with their hosts (6). The current, provisional classification of crAss-like phages based on gene sharing patterns, lists ten genera (denoted I-X) in four subfamilies (Alphacrassvirinae, Betacrassvirinae, Gammacrassvirinae and Deltacrassvirinae). Genus I includes the original p-crAssphage and is by

330 far the most commonly detected and the most abundant member of the family in the human gut, at least in the Western populations (23). However, the only genus that contains a cultured representative is candidate genus VI (Betacrassvirinae) which are widespread but only moderately abundant in the human gut. Members of this genus were isolated from human faeces (ФcrAss001), wastewater effluent (DAC15 and DAC17), and sea water (Ф14:2), infecting B. intestinalis (9), B.

335 thetaiotaomicron (17) and C. baltica, respectively (22). All these phages were able to lyse target cultures at least partially and produce spots and plaques in a semi-solid agar overlay assay. Phage of genus I and other genera common in the human gut could be grown in vivo after transfer into oligoxenic mice or after faecal microbial transplantation of crAssphage-rich microbiota into human patients, and ex vivo as part of a native faecal community in a bioreactor, but have not as yet been

340 isolated in pure bacterial cultures $(6,7,16,26)$.

We hypothesize that crAss-like phage-host systems encompass a range of behaviours, from one resembling virulent phages that produce plaques but whose replication in pure host cultures and complex microbial communities is typically supported at lower levels; to one similar to temperate phage that produce no plaques but persist in host populations at very high levels. It is notable that,

345 regardless of the degree of virulence of a crAss-like phage, their stable and high-level persistence in vitro and in vivo has no obvious detrimental effect on the population densities or fitness of their bacterial host. This phenomenon is generally consistent with a temperate mode of replication, but we conclude from our analysis of ФcrAss001, DAC15 and DAC17 that these viruses are nonlysogenic. Specifically, (i) no cases of related prophages have ever been observed in public

350 databases of bacterial genomes; (ii) no clear lysogeny modules could be identified their genomes; and (iii) lysogens or pseudolysogens could not be obtained experimentally.

Observations of long-term persistence of virulent bacteriophages in the mammalian gut are not unprecedented. Experiments in monoxenic mice revealed that administration of virulent phage fails to lower the levels of target $E$. coli bacteria and often leads to continuous shedding of high phage

355 levels in faeces $(27,28)$. Alternative mechanisms of non-lethal, persistent forms of infection were described in virulent phages, including the carrier state and unstable pseudolysogeny in 
Campylobacter jejuni, Salmonella typhimurium (phage P22) and Pseudomonas aeruginosa (29-31). In C. jejuni, virulent bacteriophages CP8 and CP30A have been shown to maintain equilibrium with their hosts for many generations and to persist intracellularly in an episomal form without

integration into the host chromosome. It has also been demonstrated that the carrier state can have a profound effect on bacterial host physiology, such as a major acquisition of self-targeting CRISPR spacers, decreased fitness, loss of colonisation potential and motility in C. jejuni (32). More recently a carrier state was described in P. aeruginosa infected with ssRNA levivirus LeviOr01 (33). Infection with LeviOr01 caused dissociation of the sensitive host into two sub-populations: a small

365 subset of phage-carrying superinfection immune cells, spontaneously and continuously releasing phage progeny, and a larger subset of uninfected non-carrier cells. Together, previous observations suggest that unconventional forms of phage infection span a wide taxonomic variety of bacteria and levels of complexity of phages (from Leviviridae containing only four genes in their genomes, to Myoviridae with hundreds of genes).

370 The genus Bacteroides comprises strictly anaerobic Gram-negative non-sporeforming bacteria, many of which are predominant members of the human gut microbiome and are highly adapted to the gut environment (34). Genome plasticity, extensive regulation of gene expression and high adaptability to changes in their environment are characteristic features of this bacterial group (35). Phase variation of surface structures resulting from DNA inversions catalysed by serine and

375 tyrosine recombinases has been implicated as an important mechanism in Bacteroides for adaptation to environmental change, improved fitness, and efficient colonisation of the mammalian gut (20, 24, 36-38). Recently, phase variation of multiple surface structures including CPS, S-layer lipoproteins, TonB-dependent nutrient receptors and OmpA-like proteins, has also been implicated in dynamic switching of diverse phage resistance/sensitivity patterns in B. thetaiotaomicron (14).

380 Furthermore, crAss-like phages DAC15 and DAC17 were shown to preferentially infect a phase variant of $B$. thetaiotaomicron expressing a specific type of CPS (17). Recent modelling with phase variable phage receptor in Haemophilus influenzae demonstrated the role of phase variation in bacterial "herd immunity", a phenomenon in phage-host population dynamics where "protective quarantining" of one third of the host cells already confers significant protection against phage

385 attack to the whole population (39).

In this study we shed some light on the mechanisms underpinning persistence of crAss-like phages using the $\Phi$ crAss001-B. intestinalis pair as a model. We observed that, similarly to metagenomic findings from the human gut $(6,8)$, a monoxenic mouse model was able to support long term and stable persistence of $\Phi$ crAss001. Bacterial clones recovered from colonised mice showed varied

390 degree of resistance to phage, but no obvious cost with regards to bacterial population density was associated with phage colonisation. We propose that that at least two separate mechanisms operating simultaneously are responsible for observed persistence in vitro: (i) dynamic and reversible acquisition of phage resistance in the host population, dependent on phase variation of CPS; (ii) delayed release or progeny from infected cells, resulting in a pseudolysogenic-like or 395 carrier state phenotype. (Fig. 5).

Sustained phage attack resulted in either switching off the PVR9 CPS locus, implicating this structure in phage adsorption, or increased expression of alternative CPS's (PVR7, PVR8, PVR11 and PVR12) with possible protective effects. We propose that the dynamic switching between different CPS types maintains an equilibrium of sensitive and resistant cells, allowing for restricted ("quarantined") phage replication and enabling long term phage persistence and herd immunity in 
the bacterial population. While phage attack provides an obvious selective force driving switching to resistance states, the force driving a return to sensitivity remains unidentified. In complex microbiota conditions, expression of CPS protective against one phage could make cells sensitive to another phage present in the same environment (14), creating an equilibrium of CPS expression

405 states driven by opposing phages. In monoculture, however, such diverse phage selective forces are absent, making the metabolic cost of expression of a particular protective CPS an obvious reason for switching it off. Availability of a single-CPS mutant panel in B. thetaiotaomicron prompted us to check whether persistence of DAC15 and DAC17 is still possible despite inability of the strain to evade phage by swapping its CPS types. Indeed, not only persistence in such strain was still possible, but actually occurred at higher levels than in the WT.

We hypothesise that a second mechanism of phage persistence operates in parallel with herd immunity. This is based on the low apparent burst size (despite progeny counts of $>50$ per cell under the EM), multiple sequential smaller bursts and cells carrying phage progeny without lysing in a timely manner, the high expression of mainly early genes (regulatory proteins) and some of the

415 middle genes (replication functions) in broth cultures of $B$. intestinalis in the presence of phage. Several explanations are possible: (a) carrier state, persistence in the form of fully formed or incompletely formed viral particles in the host cytoplasm; (b) unstable pseudolysogeny, persistence of phage genomes in an episomal form; (c), inhibition of phage reproduction by cellular mechanisms and curing of infected cells. Interestingly, overwhelming the cells with high MOI was enough to overcome this mechanism of gradual release of progeny in B. thetaiotaomicron but not in B. intestinalis.

We suggest that the interplay between phase variation of phage receptors and the carrier state infection enable highly synchronised replication of a crAss-like phage with its host at a population level, and leads to an equilibrium of phage/host ratio maintained over multiple generations.

425 It has been proposed that high availability of bacterial prey in aquatic environments and at mucosal surfaces selects for temperate bacteriophage lifestyles and lysogenic mode of replication. Such persistent infection is highly beneficial for the phage and is at least 'affordable' (if not also beneficial) to the host $(40,41)$. This ecological model has become known as "piggyback-thewinner", as opposed to the "kill-the-winner" model (41). The mammalian gut presents an example

430 of one of the most densely populated microbial communities, with Bacteroides being one of its most abundant and temporally stable bacterial genera $(6,42,43)$. Our expectation was that the majority of bacteriophages in such an environment would be temperate, "piggybacking” on the ecological success of their bacterial hosts (44-46). At the same time our recent metagenomic analysis highlighted the prevalence of apparently virulent bacteriophages in the healthy core virome

$435(6,10)$. One could hypothesise that crAss-like phages, incapable of classical lysogeny and comprising a large part of these metagenomic sequences, employ alternative life cycle strategies, such as the one highlighted in this this study in order to benefit from "piggyback-the-winner" dynamics.

While the ecological benefits of carrier state infection are obvious for the phage, we can expect that 440 the bacterial host party should also derive certain benefits from this interaction. In addition to exerting a constant selective pressure leading to host diversification through phase variation and point mutations and an improved overall fitness, persistent phage can participate in lateral gene transfer, provide protection from incoming competitor strains and superinfection immunity against cognate phages. 


\section{Acknowledgements}

This research was conducted with the financial support of Science Foundation Ireland (SFI) under Grant Number SFI/12/RC/2273, a Science Foundation Ireland's Spokes Programme which is cofunded under the European Regional Development Fund under Grant Number SFI/14/SP

450 APC/B3032, and a research grant from Janssen Biotech, Inc. Authors wish to thank Frances O’Brien and Tara O'Driscoll (UCC) for their technical help with germ-free mouse experiment.

\section{Author contributions}

Conceptualization, A.N.S. and C.H.; Methodology, A.N.S., E.V.K., N.S., A.J.H., C.Heuston and

455 D.S.; Software, A.N.S.; Formal analysis, A.N.S., E.V.K., D.S. and C.Hill; Investigation, A.N.S., E.V.K., C.Heuston and S.S.; Resources, A.J.H., D.S. and C.Hill; Writing - original draft, A.N.S. and E.V.K.; Writing - review and editing, A.N.S., E.V.K., A.J.H., C.Heuston , D.S., R.P.R. and C.Hill; Supervision, D.S., C.Hill and R.P.R.; Project administration, C.Hill; Funding acquisition, C.Hill and R.P.R.

\section{Declaration of interests}

The authors declare that they have no competing interests.

\section{Data and code availability}

465 All data needed to evaluate the conclusions in the paper are present in the paper and/or the Supplementary Materials. Further information and requests for data and resources should be directed to and will be fulfilled by Dr. Andrey Shkoporov (andrey.shkoporov@ucc.ie). Raw sequencing data are available from NCBI databases under BioProject PRJNA678472. Raw numerical data and images used to produce Fig. 1-4 are available from the Supplementary Dataset (https://figshare.com/s/c3485a7633d3632579ca).

\section{Materials availability}

Phage ФcrAss001 and its host B. intestinalis APC919/174 are available from DSMZ culture collection under catalogue numbers DSM 109066 and DSM 108646 respectively. Phages DAC15

475 and DAC17, as well as B. thetaiotaomicron VPI-5482 capsule mutants are available on request from Prof. A.J. Hryckowian.

\section{Figure legends}

480 Figure 1. Long term persistence of crAss-like phages in the gut microbiome and in vitro. (a), Persistence of 11 crAss-like phage genomes (belonging to candidate genera I,II,V,VI,VII,X according to Guerin et al. [2018] taxonomy) in faecal VLP fractions of 10 healthy adult individuals over a period of 12-26 months (6); (b), Persistence of $\Phi$ crAss001 in the gut of C57BL/6NTac mice $(\mathrm{n}=6)$ mice colonised with $\Phi \mathrm{crAss} 001 / B$. intestinalis APC919/174 mixture at an MOI=1; the

485 control group of mice received oral gavage of $B$. intestinalis only; (c), replication of $\Phi c r$ Ass001 in exponentially growing culture of $B$. intestinalis (OD600 $=0.3$ at infection with an MOI=1) followed by four daily transfers of phage/host co-culture in fresh broth; bacterial and free phage counts were obtained by plating and plaque assays respectively; (d), VMR determined using shotgun 
metagenomic sequencing in the same phage/host co-cultures as in (c), with additional samples collected after 18 and 23 daily transfers.

Figure 2. Resistance of $B$. intestinalis APC919/174 to ФcrAss001 is reversible and associated with capsular polysaccharide alterations and loss of phage adsorption. (a), spotting of $4 \times 10^{7}$ pfu of ФcrAss001 on B. intestinalis APC919/174 agar overlay results in turbid clearing zone; (b-f), 495 TEM $(x 9,900-105,000)$ of ultra-thin sections $(80 \mathrm{~nm})$ of samples taken from the centre of the spot shows the presence of un-infected cells amongst lysed cell debris, infected cells with empty virions ("ghosts") still attached, and cells in the process of division with phage progeny visible inside of them; (g) and (h), soft agar overlays inoculated with the same number of B. intestinalis CFU in the absence and presence of the excess of phage, respectively; (i), phenotypic dissociation of colonies

500 of phage-resistant clone 8, (8W, “white” - phage-sensitive subclone; 8T, “transparent” - phageresistant subclone); (j), kinetics of ФcrAss001 adsorption to B. intestinalis phage resistant and sensitive clones; vertical axis, concentration of phage in the supernatant; horizontal axis, time passed after addition of phage; $R$, resistant clones $(n=5)$; Rev, revertant sensitive sub-clones derived from one of the resistant clones; values are mean \pm SD from three independent experiments; (k-l),

505 ultrathin section TEM visualisation $(\mathrm{x} 60,000)$ of altered cell surface morphology in phage-resistant clone 8T, compared to phage-sensitive WT strain; notable is the production of either loose or compact capsule; cm, cytoplasmic membrane; om, outer membrane.

Figure 3. Structure and transcriptional control of five phase-variable capsular polysaccharide (CPS) operons (PVR7, PVR8, PVR9, PVR11, PVR12) in B. intestinalis APC919/174. (a), host transcriptional response in a B. intestinalis APC919/174-ФcrAss001 serial broth co-culture (24$120 \mathrm{~h}$ in vitro persistence) experiment, analysed using RNAseq (values are log2 fold gene transcript expression change relative to the un-infected control from two independent experimental runs); clone-8T, phage resistant derivative of the parental strain; clone-8W, spontaneous phage-sensitive

515 revertant clone; only genes with significantly changed expression ( $\mathrm{p}<0.01$ in DESeq2, $\mathrm{n}=826)$ are shown; genes, associated with phase-variable genomic regions (PVR) are marked with colour bars on top; (b), structure of phase variable operons, encoding five different CPS; protein sequence homologies (tBLASTx) between gene products are shown as coloured parallelograms; two-sided arrows and ON/OFF labels mark positions of invertible promoters; (c), consensus sequence of the

520 regions flanking invertible promoters in CPS biosynthesis loci; approximate transcription start was identified though analysis of RNAseq data; stacked area charts show RNAseq read coverage of regions adjacent to the promoter in four CPS loci; (d-e), fraction or Illumina concordantly-aligned read pairs supporting orientation of invertible promoters in either ON or OFF directions; WT, wild type strain; clones 8T, PhR1-9, phage-resistant derivatives; 8W, phage-sensitive revertant;

$525 \mathrm{P} 18 \mathrm{~d} / \mathrm{P} 23 \mathrm{~d}$, samples in (e) were taken from the long term in vitro persistence experiment (also see Fig. S3 for similar data on the long term in vivo persistence).

\section{Figure 4. Transcriptional and morphological presentation of ФcrAss001 infection in a one-} step growth experiment. (a), transcription of phage genome 0-120 min after infection revealed using stranded RNAseq; positive and negative strand ORFs and RNAseq coverage levels are shown in blue and red, respectively; plots are representative of two independent experiments; (b), time course of transcriptional responses of host and phage genomes (values are log2 fold gene transcript 
expression change relative to the previous time point from two independent experimental runs); only genes with significantly different expression of sense DNA strand between any of the successive time points ( $\mathrm{p}<0.05$ in DESeq2, $\mathrm{n}=372$ ) are shown; $(\mathbf{c})$, time-course of morphological changes in $B$. intestinalis cells infected with ФcrAss001, ultrathin section TEM (x43,000); notable are intracellular phage progeny and possible "phage scaffolds" in the early stages of virion assembly; (d-e), enumeration of per capita phage progeny produced in a one-step growth experiment by $B$. intestinalis cells infected with $\Phi$ crAss001 [MOI=1 in panel (d), MOI=10 in panel (e)]; (f), intact cells after first phage burst (150 min), still showing hallmark features of the early and middle infection stages $(\mathrm{x} 6,000)$.

Figure 5. Model of perpetuated replication of crAss-like phage in Bacteroides cultures. (Created using BioRender.com). See Discussion for details.

\section{Materials and methods}

\section{Bacteriophage $\Phi$ crAss001 and related viruses}

The host strain B. intestinalis APC919/174 was propagated anaerobically on Fastidious Anaerobe Broth (FAB, Neogen, UK) and agar (FAA) at $37^{\circ} \mathrm{C}$ as described before (9). To generate high titer phage lysates, early log phase culture $\left(\mathrm{OD}_{600}=0.2 ; \sim 2 \times 10^{8} \mathrm{cfu} \mathrm{ml}^{-1}\right)$ were infected with $\Phi \mathrm{crAss} 001$ at an MOI of 1 . Cultures were left to grow overnight at $37^{\circ} \mathrm{C}$ in anaerobic jars, centrifuged for 15 min at 5,200 g and $4^{\circ} \mathrm{C}$ and filtered through $0.45 \mu \mathrm{m}$ polyethersulfone (PES) syringe-mounted filter membranes. Plaque and spot assays throughout different experiments were performed after filtering

$5551 \mathrm{~mL}$ of phage/bacteria co-cultures, performing serial dilutions and combining $100 \mu \mathrm{l}$ of them with $300 \mu \mathrm{l}$ of a fresh APC919/174 overnight culture in 0.4\% bacto agar overlays. Incubations were performed anaerobically at $37^{\circ} \mathrm{C}$. Bacteriophages DAC15 and DAC17 and their host strains Bacteroides thetaiotaomicron VPI-5482 (WT and $c p s 3^{+}$) were propagated in the same conditions as B. intestinalis/ФcrAss001.

\section{Germ-free mouse model}

The germ-free animal experiment was performed in accordance with the European Communities Council Directive 2010/63/EU under an authorisation issued by the Health Products Regulatory Authority (HPRA, Ireland), with approval from the Animal Experimentation Ethics Committee 565 (AEEC) of University College Cork. Twelve male 13 week old (26-29 g) germ-free C57BL/6N mice (RRID:MGI:5651595, original breeding stock from Taconic, USA; bred in house) were housed in groups of 2-3 mice in HEPA filtered individually ventilated cages to maintain germ-free status. All dosing and faecal sampling occurred under sterile conditions in a biosafety cabinet to ensure there was no confounding colonisation. Group $1(n=6)$ were orally dosed on three

570 successive days with $100 \mu \mathrm{l}$ of phage/bacteria suspension $\left(10^{9} \mathrm{cfu}\right.$ of bacteria, $2 \times 10^{8}$ pfu of phage per gavage). Group 2 were orally dosed with bacteria only $\left(10^{9} \mathrm{cfu}\right.$ per gavage). Faecal samples were collected for 146 days after the first dose (days 3, 6-10, 14, 17, 24, 31, 38, 45, 59, 87, 93, 101, $108,115,121,129,136,146)$ and subjected to plating and plaque assays for enumeration of bacteria and phage (Fig. 1b). On the last day of the experiment animals were humanely euthanised by

575 cervical dislocation. 


\section{In vitro experiments with crAss-like phages}

Phage adsorption experiments (Fig. 2j) were conducted in aerobic conditions at room temperature on cells from an overnight Bacteroides culture $\left(10 \mathrm{~mL}\right.$, optical density adjusted to $\left.\mathrm{OD}_{600}=0.2\right)$, triple washed with growth medium and resuspended in the same media volume before the experiment. Phage was applied at an MOI=1, aliquots were removed, filtered as described above and subjected to plaque assays 5 and 20 min after addition of phage.

Efficiency of bacterial plating (EOP) in the presence of phage (Fig. 2g-h) was conducted as follows: Serial tenfold dilutions of overnight Bacteroides cultures were inoculated into $3 \mathrm{~mL}$ of 585 semi-solid FAA ( $0.4 \%$ agar) with or without addition of $100 \mu \mathrm{L}$ high-titre phage $\left(\sim 10^{10} \mathrm{pfu} \mathrm{mL}^{-1}\right)$ and poured onto $100 \mathrm{~mm}$ diameter FAA agar plates. Efficiency of lysogeny was determined as a percentage of colonies observed on phage-containing overlays relative to the total counts on negative control overlays after 48 hours of anaerobic incubation at $37^{\circ} \mathrm{C}$.

In vitro phage persistence experiment (Figs. 1c-d, 3a, 3d, S5d) was performed as follows: ten

590 millilitre liquid cultures $(\mathrm{n}=3)$ of Bacteroides were infected at $\mathrm{OD}_{600}=0.2$ and $\mathrm{MOI}=1$, and incubated overnight. The co-culture was then transferred daily, for five days in fresh FAB medium at 1:50 ratio.

One-step growth curves (Fig. 4d-e, S5e-g) were built as follows: early logarithmic phase $\left(\mathrm{OD}_{600}=0.2\right)$ cultures of Bacteroides $(20 \mathrm{~mL})$ were infected at an MOI of 1 or 10 for $5 \mathrm{~min}$ at room

595 temperature, followed by centrifugation at 5,200 g, $4^{\circ} \mathrm{C}$ for $15 \mathrm{~min}$, removal of supernatant and resuspending of the infected cells in fresh FAB medium. Incubation was continued anaerobically at $37^{\circ} \mathrm{C}$ for further $225 \mathrm{~min}$ with removal of $1 \mathrm{~mL}$ samples every $15 \mathrm{~min}$. Samples were filtered through $0.45 \mu \mathrm{m}$ pore PES filters and subjected to standard plaque assays with appropriate dilutions. Efficiency of centre of infection (EOCI) was determined by doing plaque assays with infected cells at the same time intervals but without filtration step.

\section{Transmission electron miscroscopy}

Ultrathin section TEM of Bacteroides cells grown in broth cultures (Fig. 2k-l) or embedded in semi-solid agar (Figs. 2b-f, 4c, 4f, S4) were performed as follows. Pellets from $1 \mathrm{~mL}$ broth cultures or agar overlay fragments ( $100 \mu \mathrm{L}$ volume) were immediately fixed and stored in $1 \mathrm{~mL}$ of $2 \%(\mathrm{v} / \mathrm{v})$ glutaraldehyde, 1.5\% (w/v) parafolmaldehyde, $75 \mathrm{mM}$ Tris- $\mathrm{HCl} \mathrm{pH} 7.5$ solution. Samples were post fixed with 1\% Osmium tetroxide in 0.1 M Sorenson's phosphate buffer for 1 hour at room temperature. A series of graded dehydrations were performed with increasing ethanol concentrations (30\%, 50\%, 70\%, 100\%; cells from broth cultures were pelleted by centrifugation between steps).

610 Dehydrated samples were embedded in Epon resin. Briefly, dehydrated samples were transferred into 100\% acetone and then 50:50 acetone:Epon resin for a minimum of 1 hour. Samples were then put in $100 \%$ Epon resin at $37^{\circ} \mathrm{C}$ for 2 hours. Resin was polymerised at $60^{\circ} \mathrm{C}$ for a minimum of 24 hours. Embedded samples were sectioned at $80 \mathrm{~nm}$ thickness onto copper grids using a Leica UC6 ultramicrotome. Samples were stained with $2 \%$ uranyl acetate for 20 minutes and $3 \%$ lead citrate

615 for 5 minutes. Grids were then imaged on a FEI Tecnai 120 at $120 \mathrm{kV}$ accelerating voltage. TEM of purified phage particles (Figs. S5a-b) was performed as described before (9).

\section{Shotgun sequencing and analysis of bacterial genomes}

Shotgun sequencing on Illumina platform was performed for parental strain APC919/174 (Figs. 3b, 620 3e, S1, S2), resistant clones PhR1-9, 8T and spontaneous sensitive revertant derivative (8W) of the 
latter (Figs. 3e, S1, S2), as well as samples from in vitro phage persistence experiment and faecal samples from mice colonized with $\Phi$ crAss001/B. intestinalis phage-host pair (Figs. 3d, S3). BioSample accession number are SAMN16802447-SAMN16802559 and SAMN16803214. Genomic DNA was extracted from bacterial cultures and phage-host co-cultures using DNeasy Blood \& Tissue Kit (Qiagen) in accordance with the manufacturer's Gram-negative bacteria protocol. Faecal DNA was extracted using QIAamp Fast DNA Stool Mini Kit (Qiagen). Concentration of DNA was determined using the Qubit dsDNA HS kit and the Qubit 3 fluorometer (Invitrogen/ThermoFisher Scientific). One hundred nanograms of purified DNA sample were sheared with M220 Focused-Ultrasonicator (Covaris) applying the 350 bp DNA fragment length

630 settings (peak power $50 \mathrm{~W}$, duty factor $20 \%$, 200 cycles per burst, total duration of 65 s). Random shotgun libraries were prepared from genomic DNA using TruSeq Nano Library Preparation Kit (Illumina, cat \#20015964) with dual-indexing following the standard manufacturer's protocol. Paired-end sequencing with $2 \times 150$ nt chemistry was performed on HiSeq 4000 platform at Eurofins Genomics.

635 The quality of raw sequences (SRA database records SRR13062103-SRR13062215) was assessed using FastQC v0.11.5. TruSeq adapters were removed with cutadapt v2.4. To trim sequences and remove low quality reads, Trimmomatic v0.36 was applied using the following parameters: 'SLIDINGWINDOW:4:20 MINLEN:60 HEADCROP:10', yielding 2.2-9.7 million reads per sample (median 5.9 million).

640 For long read Oxford Nanopore sequencing genomic DNA from APC919/174, 8T, 8W, Phr5 and Phr5-1 (sensitive derivative of the resistant clone Phr5) was extracted as follows. Cells from two $\mathrm{mL}$ of overnight cultures were collected by centrifugation at 5,000 $\mathrm{g}$ for $10 \mathrm{~min}$, washed by resuspending in $1 \mathrm{~mL}$ of TES (50 mM NaCl, $100 \mathrm{mM}$ Tris-HCl, $70 \mathrm{mM}$ disodium EDTA, $\mathrm{pH}$ 8.0) and re-centrifuged. After resuspending cell pellets in $0.4 \mathrm{~mL}$ of TES supplemented with $25 \% \mathrm{w} / \mathrm{v}$

645 sucrose and $30 \mathrm{mg} \mathrm{mL}^{-1}$ of lysozyme, $50 \mathrm{U}$ of RNase I (Fisher Scientific) were added and samples were incubated at $42^{\circ} \mathrm{C}$ for 1 hour. Cells were then lysed by addition of $20 \mu \mathrm{L}$ of $10 \%(\mathrm{w} / \mathrm{v})$ sarkosyl and $5 \mu \mathrm{L}$ of $20 \mathrm{mg} \mathrm{mL}^{-1}$ proteinase $\mathrm{K}$ solutions with incubation at $37^{\circ} \mathrm{C}$ until complete clearing of the lysate (30-60 min). The obtained lysates were diluted by adding $350 \mu \mathrm{L}$ of TES and $16 \mu \mathrm{L}$ of $5 \mathrm{M} \mathrm{NaCl}$ and gently extracted twice with equal volume of 25:24:1

650 phenol:chloroform:isoamyl alcohol mix with room temperature centrifugation at 8,000 g for $5 \mathrm{~min}$. This was followed by a similarly done extraction by an equal volume of chloroform. Finally, aqueous phase was gently mixed with 2 volumes of $96 \%$ ethanol and DNA was pelleted by centrifugation at 8,000 g, $4^{\circ} \mathrm{C}$ for $15 \mathrm{~min}$. Pellets were rinsed with $1 \mathrm{~mL}$ of $70 \%$ ethanol, air dried and resuspended in $50 \mu \mathrm{L}$ TE (10 mM Tris-HCl, $0.1 \mathrm{mM}$ disodium EDTA, pH 8.0) by overnight

655 incubation at $4^{\circ} \mathrm{C}$.

Long-read sequencing libraries (SRR13062400, SRR13062401, SRR13062402, SRR13062326, SRR13062327) were prepared using Oxford Nanopore Rapid Barcoding Kit (SQK-RBK004) and pooled. The pooled library was then loaded onto R9 version flowcell (FLO-MIN106D) and sequenced in a MinION sequencer (Oxford Nanopore) for 48 hours. Sequencing data was then

660 basecalled, quality-filtered and de-multiplexed using Guppy v3.1.5. This yielded a total of 299,317 reads of 1,882 $\pm 4,697 \mathrm{nt}$ length (median $\pm \mathrm{IQR}$ ) for APC919/174, 27,348 reads of 11,268 $\pm 24,836 \mathrm{nt}$ length for $8 \mathrm{~T}, 57,259$ reads of 10,529 $\pm 21,955 \mathrm{nt}$ length for $8 \mathrm{~W}, 31,238$ reads of 13,673 $\pm 32,494 \mathrm{nt}$ length for Phr5, and 6,547 reads of 4,207 $\pm 16,442$ nt length for Phr5. 
Trimmed and filtered Illumina reads and raw Oxford Nanopore reads were used for hybrid assemblies of the genomes of strains APC919/174, 8T and 8W using SPAdes assembler v3.13.0 in 'careful' mode. This resulted in complete circular contigs of 5,785,761 bp, 5,785,768 bp and 5,785,766 bp respectively. Genomes were annotated using RASTtk and PGAP pipelines and deposited in NCBI Genbank under accessions numbers CP041379, CP064941 and CP064940. Completed genomes were aligned using progressive MAUVE algorithm (version 2015-02-13) to

670 identify regions of major recombination (Fig. S1).

For detection of PVR promoter inversions and variant analysis in the in vitro co-culture and mouse colonisation experiments (Figs. 3d-e, S3b) paired Illumina reads were mapped to the assembled APC919/174 as a reference. Reads were aligned using bowtie2 v2.3.4.1 in the end-to-end mode. Alignments were sorted, indexed and converted into counts tables using samtools v1.7.

675 For detection of genomic recombination on a single read level, sufficiently long Oxford Nanopore reads (> $1000 \mathrm{nt}$ ) were aligned against APC919/174 reference genome using BLASTn v2.10.0 with e-value cut-off of 1e-20. Individual alignments were retained only if length was $>200 \mathrm{nt}$ with identity of $>90 \%$. Next, reads were identified containing internally inverted alignments or blocks of aligned sequence corresponding to coordinates in the reference genome shifted $>200 \mathrm{nt}$ relative to other aligned blocks inside the same read. Such blocks of inconsistently aligned sequence were deemed as evidence of recombination events dynamically unfolding inside an individual clonal culture. Starting coordinates of these misaligned blocks were then plotted against the APC919/174 reference genome in a histogram with $1000 \mathrm{nt}$ long bins and hotsposts of recombination were identified (Fig. S2).

685

\section{RNAseq procedures}

Transcriptomic analysis of $\Phi$ crAss001/B. intestinalis co-cultures was conducted for in vitro phage persistence experiment (Fig. 3a, 3c; BioSamples SAMN16809412-SAMN16809423) and one-step growth experiment (Fig. 4a-b; BioSamples SAMN16810643-SAMN16810654).

690 One millilitre culture aliquots were pelleted by centrifugation for $3 \mathrm{~min}$ at 17,000 g, anaerobically. Pellets were immediately lysed using $1 \mathrm{~mL}$ of TRIzol reagent (ThermoFisher Scientific). Total bacterial RNA was extracted using the standard manufacturers protocol. Extracted RNA $(20 \mu \mathrm{L})$ was treated with 2U TURBO DNase (ThermoFisher Scientific), further purified using RNeasy Mini Kit (Qiagen) and assayed for RNA integrity and quantity on Agilent Bioanalyzer using 6000 RNA

695 Nano Kit.

Stranded RNA-seq libraries were prepared from $1 \mu \mathrm{g}$ total RNA using ScriptSeq v2 Complete kit for bacteria (Epicentre) and sequenced on Illumina NovaSeq 6000 platform at GENEWIZ. Read quality checks, filtration and trimming was performed as described above in Shotgun sequencing and analysis of bacterial genomes.

700 Reads were then mapped to ФcrAss001 and B. intestinalis APC919/174 reference genomes (NCBI accession numbers MH675552 and CP041379 respectively) using bowtie2 v2.3.4.1 in the end-toend mode. Alignments were sorted by DNA strand and indexed using samtools v1.7. Samtools 'mpileup' command was used to calculate read coverage per nucleotide position in both genomes. HTSeq framework v0.6.1.p1 was used to calculate aligned read counts per feature with default

705 parameters. Read counts and coverage tables were then imported into R environment using data.table library v1.12.8 and compared between time points using DESeq2, using Wald test and parametric fit. 


\section{Quantification and statistical analysis}

710 Bacterial colonisation levels in vivo in the presence or absence of phage (Fig. 1b) were compared using Kruskal-Wallis test with FDR correction and were all found to be insignificantly different, except for days 31 and 38 ( $<$ 0.05). Adsorption of crAss001 to sensitive and resistant bacterial variants (Fig. 1b) was compared using Kruskal-Wallis test with Bonferroni correction and was found significantly different ( $\mathrm{p}<0.05$ ). Analysis of differentially abundant transcripts (Figs. 3a, 4b)

715 was performed using DESeq2. Only genes showing significant change $(\mathrm{p}<0.001$ for host genes in in vitro persistence experiment, Fig. 3a, and $p<0.05$ for both phage and host genes in one step growth experiment, Fig. $\mathbf{4 b}$ ) between any of the consecutive time points are displayed. Bacterial genes differentially affected by mutations between the two groups of animals (phage and no phage, Fig. S3b) in mouse colonisation experiment were selected using exact Fisher's test $(p<0.05$ with FDR correction).

\section{References}

1. N. Yutin, S. Benler, S. A. Shmakov, Y. I. Wolf, I. Tolstoy, M. Rayko, D. Antipov, P. A. Pevzner, E. V. Koonin, bioRxiv, in press, doi:10.1101/2020.07.20.212944.

2. T. P. Honap, K. Sankaranarayanan, S. L. Schnorr, A. T. Ozga, C. Warinner, C. M. L. Jr, Biogeographic study of human gut-associated crAssphage suggests impacts from industrialization and recent expansion. PLOS ONE. 15, e0226930 (2020).

3. E. V. Koonin, N. Yutin, The crAss-like Phage Group: How Metagenomics Reshaped the Human Virome. Trends in Microbiology. 28, 349-359 (2020).

4. N. Yutin, K. S. Makarova, A. B. Gussow, M. Krupovic, A. Segall, R. A. Edwards, E. V. Koonin, Discovery of an expansive bacteriophage family that includes the most abundant viruses from the human gut. Nature Microbiology. 3, 38-46 (2018).

5. R. A. Edwards, A. A. Vega, H. M. Norman, M. Ohaeri, K. Levi, E. A. Dinsdale, O. Cinek, R. K. Aziz, K. McNair, J. J. Barr, K. Bibby, S. J. J. Brouns, A. Cazares, P. A. de Jonge, C. Desnues, S. L. Díaz Muñoz, P. C. Fineran, A. Kurilshikov, R. Lavigne, K. Mazankova, D. T. McCarthy, F. L. Nobrega, A. Reyes Muñoz, G. Tapia, N. Trefault, A. V. Tyakht, P. Vinuesa, J. Wagemans, A. Zhernakova, F. M. Aarestrup, G. Ahmadov, A. Alassaf, J. Anton, A. Asangba, E. K. Billings, V. A. Cantu, J. M. Carlton, D. Cazares, G.-S. Cho, T. Condeff, P. Cortés, M. Cranfield, D. A. Cuevas, R. De la Iglesia, P. Decewicz, M. P. Doane, N. J. Dominy, L. Dziewit, B. M. Elwasila, A. M. Eren, C. Franz, J. Fu, C. Garcia-Aljaro, E. Ghedin, K. M. Gulino, J. M. Haggerty, S. R. Head, R. S. Hendriksen, C. Hill, H. Hyöty, E. N. Ilina, M. T. Irwin, T. C. Jeffries, J. Jofre, R. E. Junge, S. T. Kelley, M. Khan Mirzaei, M. Kowalewski, D. Kumaresan, S. R. Leigh, D. Lipson, E. S. Lisitsyna, M. Llagostera, J. M. Maritz, L. C. Marr, A. McCann, S. Molshanski-Mor, S. Monteiro, B. Moreira-Grez, M. Morris, L. Mugisha, M. Muniesa, H. Neve, N. Nguyen, O. D. Nigro, A. S. Nilsson, T. O’Connell, R. Odeh, A. Oliver, M. Piuri, A. J. Prussin Ii, U. Qimron, Z.-X. Quan, P. Rainetova, A. Ramírez-Rojas, R. Raya, K. Reasor, G. A. O. Rice, A. Rossi, R. Santos, J. Shimashita, E. N. Stachler, L. C. Stene, R. Strain, R. Stumpf, P. J. Torres, A. Twaddle, M. Ugochi Ibekwe, N. Villagra, S. Wandro, B. White, A. Whiteley, K. L. Whiteson, C. Wijmenga, M. M. Zambrano, H. Zschach, B. E. Dutilh, Global phylogeography and ancient evolution of the widespread human gut virus crAssphage. Nature Microbiology. 4, 1727-1736 (2019).

6. A. N. Shkoporov, A. G. Clooney, T. D. S. Sutton, F. J. Ryan, K. M. Daly, J. A. Nolan, S. A. McDonnell, E. V. Khokhlova, L. A. Draper, A. Forde, E. Guerin, V. Velayudhan, R. P. Ross, C. 
Hill, The Human Gut Virome Is Highly Diverse, Stable, and Individual Specific. Cell Host \& Microbe. 26, 527-541.e5 (2019).

7. L. A. Draper, F. J. Ryan, M. K. Smith, J. Jalanka, E. Mattila, P. A. Arkkila, R. P. Ross, R. Satokari, C. Hill, Long-term colonisation with donor bacteriophages following successful faecal microbial transplantation. Microbiome. 6, 220 (2018).

8. B. A. Siranosian, F. B. Tamburini, G. Sherlock, A. S. Bhatt, Acquisition, transmission and strain diversity of human gut-colonizing crAss-like phages. Nature Communications. 11, 280 (2020).

9. A. N. Shkoporov, E. V. Khokhlova, C. B. Fitzgerald, S. R. Stockdale, L. A. Draper, R. P. Ross, C. Hill, $\Phi C r A s s 001$ represents the most abundant bacteriophage family in the human gut and infects Bacteroides intestinalis. Nature Communications. 9, 4781 (2018).

10. A. G. Clooney, T. D. S. Sutton, A. N. Shkoporov, R. K. Holohan, K. M. Daly, O. O’Regan, F. J. Ryan, L. A. Draper, S. E. Plevy, R. P. Ross, C. Hill, Whole-Virome Analysis Sheds Light on Viral Dark Matter in Inflammatory Bowel Disease. Cell Host \& Microbe. 26, 764-778.e5 (2019).

11. B. B. Oude Munnink, M. Canuti, M. Deijs, M. de Vries, M. F. Jebbink, S. Rebers, R. Molenkamp, F. J. van Hemert, K. Chung, M. Cotten, F. Snijders, C. J. Sol, L. van der Hoek, Unexplained diarrhoea in HIV-1 infected individuals. BMC Infectious Diseases. 14, 22 (2014).

12. M. Lourenço, L. Chaffringeon, Q. Lamy-Besnier, T. Pédron, P. Campagne, C. Eberl, M. Bérard, B. Stecher, L. Debarbieux, L. D. Sordi, The Spatial Heterogeneity of the Gut Limits Predation and Fosters Coexistence of Bacteria and Bacteriophages. Cell Host \& Microbe. 28, 390-401.e5 (2020).

13. M. Lourenço, L. De Sordi, L. Debarbieux, The Diversity of Bacterial Lifestyles Hampers Bacteriophage Tenacity. Viruses. 10, 327 (2018).

14. N. T. Porter, A. J. Hryckowian, B. D. Merrill, J. J. Fuentes, J. O. Gardner, R. W. P. Glowacki, S. Singh, R. D. Crawford, E. S. Snitkin, J. L. Sonnenburg, E. C. Martens, Phase-variable capsular polysaccharides and lipoproteins modify bacteriophage susceptibility in Bacteroides thetaiotaomicron. Nature Microbiology. 5, 1170-1181 (2020).

15. P. D. Scanlan, Bacteria-Bacteriophage Coevolution in the Human Gut: Implications for Microbial Diversity and Functionality. Trends in Microbiology. 25, 614-623 (2017).

16. B. E. Dutilh, N. Cassman, K. McNair, S. E. Sanchez, G. G. Z. Silva, L. Boling, J. J. Barr, D. R. Speth, V. Seguritan, R. K. Aziz, B. Felts, E. A. Dinsdale, J. L. Mokili, R. A. Edwards, A highly abundant bacteriophage discovered in the unknown sequences of human faecal metagenomes. Nature Communications. 5, 4498 (2014).

17. A. J. Hryckowian, B. D. Merrill, N. T. Porter, W. Van Treuren, E. J. Nelson, R. A. Garlena, D. A. Russell, E. C. Martens, J. L. Sonnenburg, Bacteroides thetaiotaomicron-Infecting Bacteriophage Isolates Inform Sequence-Based Host Range Predictions. Cell Host \& Microbe. 28, 371-379.e5 (2020).

18. D. N. Bolam, B. van den Berg, TonB-dependent transport by the gut microbiota: novel aspects of an old problem. Current Opinion in Structural Biology. 51, 35-43 (2018).

19. M. H. Foley, D. W. Cockburn, N. M. Koropatkin, The Sus operon: a model system for starch uptake by the human gut Bacteroidetes. Cell. Mol. Life Sci. 73, 2603-2617 (2016).

20. M. J. Coyne, K. G. Weinacht, C. M. Krinos, L. E. Comstock, Mpi recombinase globally modulates the surface architecture of a human commensal bacterium. PNAS. 100, 1044610451 (2003). 
21. A. V. Drobysheva, S. A. Panafidina, M. V. Kolesnik, E. I. Klimuk, L. Minakhin, M. V. Yakunina, S. Borukhov, E. Nilsson, K. Holmfeldt, N. Yutin, K. S. Makarova, E. V. Koonin, K. V. Severinov, P. G. Leiman, M. L. Sokolova, Structure and function of virion RNA polymerase of a crAss-like phage. Nature, 1-4 (2020).

22. K. Holmfeldt, N. Solonenko, M. Shah, K. Corrier, L. Riemann, N. C. VerBerkmoes, M. B. Sullivan, Twelve previously unknown phage genera are ubiquitous in global oceans. PNAS. 110, 12798-12803 (2013).

23. E. Guerin, A. Shkoporov, S. R. Stockdale, A. G. Clooney, F. J. Ryan, T. D. S. Sutton, L. A. Draper, E. Gonzalez-Tortuero, R. P. Ross, C. Hill, Biology and Taxonomy of crAss-like Bacteriophages, the Most Abundant Virus in the Human Gut. Cell Host \& Microbe. 24, 653664.e6 (2018).

24. N. T. Porter, P. Canales, D. A. Peterson, E. C. Martens, A Subset of Polysaccharide Capsules in the Human Symbiont Bacteroides thetaiotaomicron Promote Increased Competitive Fitness in the Mouse Gut. Cell Host \& Microbe. 22, 494-506.e8 (2017).

25. T. E. Rogers, N. A. Pudlo, N. M. Koropatkin, J. S. K. Bell, M. M. Balasch, K. Jasker, E. C. Martens, Dynamic responses of Bacteroides thetaiotaomicron during growth on glycan mixtures. Molecular Microbiology. 88, 876-890 (2013).

26. A. Reyes, M. Wu, N. P. McNulty, F. L. Rohwer, J. I. Gordon, Gnotobiotic mouse model of phage-bacterial host dynamics in the human gut. Proc Natl Acad Sci U S A. 110, 20236-20241 (2013).

27. D. Maura, E. Morello, L. du Merle, P. Bomme, C. L. Bouguénec, L. Debarbieux, Intestinal colonization by enteroaggregative Escherichia coli supports long-term bacteriophage replication in mice. Environmental Microbiology. 14, 1844-1854 (2012).

28. M. Weiss, E. Denou, A. Bruttin, R. Serra-Moreno, M.-L. Dillmann, H. Brüssow, In vivo replication of T4 and T7 bacteriophages in germ-free mice colonized with Escherichia coli. Virology. 393, 16-23 (2009).

29. W. Cenens, M. T. Mebrhatu, A. Makumi, P.-J. Ceyssens, R. Lavigne, R. V. Houdt, F. Taddei, A. Aertsen, Expression of a Novel P22 ORFan Gene Reveals the Phage Carrier State in Salmonella Typhimurium. PLOS Genetics. 9, e1003269 (2013).

30. L. Latino, C. Midoux, Y. Hauck, G. Vergnaud, C. Pourcel, Pseudolysogeny and sequential mutations build multiresistance to virulent bacteriophages in Pseudomonas aeruginosa. Microbiology. 162, 748-763 (2016).

31. P. Siringan, P. L. Connerton, N. J. Cummings, I. F. Connerton, Alternative bacteriophage life cycles: the carrier state of Campylobacter jejuni. Open Biology. 4, 130200 (2014).

32. S. P. T. Hooton, K. J. Brathwaite, I. F. Connerton, The Bacteriophage Carrier State of Campylobacter jejuni Features Changes in Host Non-coding RNAs and the Acquisition of New Host-derived CRISPR Spacer Sequences. Front. Microbiol. 7 (2016), doi:10.3389/fmicb.2016.00355.

33. C. Pourcel, C. Midoux, G. Vergnaud, L. Latino, A carrier state is established in Pseudomonas aeruginosa by phage LeviOr01, a newly isolated ssRNA levivirus. Journal of General Virology,. 98, 2181-2189 (2017).

34. A. G. Wexler, A. L. Goodman, An insider's perspective: Bacteroides as a window into the microbiome. Nature Microbiology. 2, 1-11 (2017).

35. L. E. Comstock, M. J. Coyne, Bacteroides thetaiotaomicron: a dynamic, niche-adapted human symbiont. BioEssays. 25, 926-929 (2003). 
36. X. Jiang, A. B. Hall, T. D. Arthur, D. R. Plichta, C. T. Covington, M. Poyet, J. Crothers, P. L. Moses, A. C. Tolonen, H. Vlamakis, E. J. Alm, R. J. Xavier, Invertible promoters mediate bacterial phase variation, antibiotic resistance, and host adaptation in the gut. Science. 363, 181-187 (2019).

37. H. Nakayama-Imaohji, H. Hirakawa, M. Ichimura, S. Wakimoto, S. Kuhara, T. Hayashi, T. Kuwahara, Identification of the Site-Specific DNA Invertase Responsible for the Phase Variation of SusC/SusD Family Outer Membrane Proteins in Bacteroides fragilis. Journal of Bacteriology. 191, 6003-6011 (2009).

38. H. Nakayama-Imaohji, K. Hirota, H. Yamasaki, S. Yoneda, H. Nariya, M. Suzuki, T. Secher, Y. Miyake, E. Oswald, T. Hayashi, T. Kuwahara, DNA Inversion Regulates Outer Membrane Vesicle Production in Bacteroides fragilis. PLOS ONE. 11, e0148887 (2016).

39. C. J. R. Turkington, A. Morozov, M. R. J. Clokie, C. D. Bayliss, Phage-Resistant PhaseVariant Sub-populations Mediate Herd Immunity Against Bacteriophage Invasion of Bacterial Meta-Populations. Front. Microbiol. 10 (2019), doi:10.3389/fmicb.2019.01473.

40. B. Knowles, C. B. Silveira, B. A. Bailey, K. Barott, V. A. Cantu, A. G. Cobián-Güemes, F. H. Coutinho, E. A. Dinsdale, B. Felts, K. A. Furby, E. E. George, K. T. Green, G. B. Gregoracci, A. F. Haas, J. M. Haggerty, E. R. Hester, N. Hisakawa, L. W. Kelly, Y. W. Lim, M. Little, A. Luque, T. McDole-Somera, K. McNair, L. S. de Oliveira, S. D. Quistad, N. L. Robinett, E. Sala, P. Salamon, S. E. Sanchez, S. Sandin, G. G. Z. Silva, J. Smith, C. Sullivan, C.

Thompson, M. J. A. Vermeij, M. Youle, C. Young, B. Zgliczynski, R. Brainard, R. A. Edwards, J. Nulton, F. Thompson, F. Rohwer, Lytic to temperate switching of viral communities. Nature. 531, 466-470 (2016).

41. C. B. Silveira, F. L. Rohwer, Piggyback-the-Winner in host-associated microbial communities. npj Biofilms and Microbiomes. 2, 16010 (2016).

42. M. Arumugam, J. Raes, E. Pelletier, D. Le Paslier, T. Yamada, D. R. Mende, G. R. Fernandes, J. Tap, T. Bruls, J.-M. Batto, M. Bertalan, N. Borruel, F. Casellas, L. Fernandez, L. Gautier, T. Hansen, M. Hattori, T. Hayashi, M. Kleerebezem, K. Kurokawa, M. Leclerc, F. Levenez, C. Manichanh, H. B. Nielsen, T. Nielsen, N. Pons, J. Poulain, J. Qin, T. Sicheritz-Ponten, S. Tims, D. Torrents, E. Ugarte, E. G. Zoetendal, J. Wang, F. Guarner, O. Pedersen, W. M. de Vos, S. Brunak, J. Doré, MetaHIT Consortium (additional Members), J. Weissenbach, S. D. Ehrlich, P. Bork, Enterotypes of the human gut microbiome. Nature. 473, 174-180 (2011).

43. R. S. Mehta, G. S. Abu-Ali, D. A. Drew, J. Lloyd-Price, A. Subramanian, P. Lochhead, A. D. Joshi, K. L. Ivey, H. Khalili, G. T. Brown, C. DuLong, M. Song, L. H. Nguyen, H. Mallick, E. B. Rimm, J. Izard, C. Huttenhower, A. T. Chan, Stability of the human faecal microbiome in a cohort of adult men. Nature Microbiology. 3, 347-355 (2018).

44. S. Minot, A. Bryson, C. Chehoud, G. D. Wu, J. D. Lewis, F. D. Bushman, Rapid evolution of the human gut virome. Proc Natl Acad Sci U S A. 110, 12450-12455 (2013).

45. J. M. Norman, S. A. Handley, M. T. Baldridge, L. Droit, C. Y. Liu, B. C. Keller, A. Kambal, C. L. Monaco, G. Zhao, P. Fleshner, T. S. Stappenbeck, D. P. B. McGovern, A. Keshavarzian, E. A. Mutlu, J. Sauk, D. Gevers, R. J. Xavier, D. Wang, M. Parkes, H. W. Virgin, Disease-specific Alterations in the Enteric Virome in Inflammatory Bowel Disease. Cell. 160, 447-460 (2015).

46. A. Reyes, M. Haynes, N. Hanson, F. E. Angly, A. C. Heath, F. Rohwer, J. I. Gordon, Viruses in the fecal microbiota of monozygotic twins and their mothers. Nature. 466, 334-338 (2010). 
a

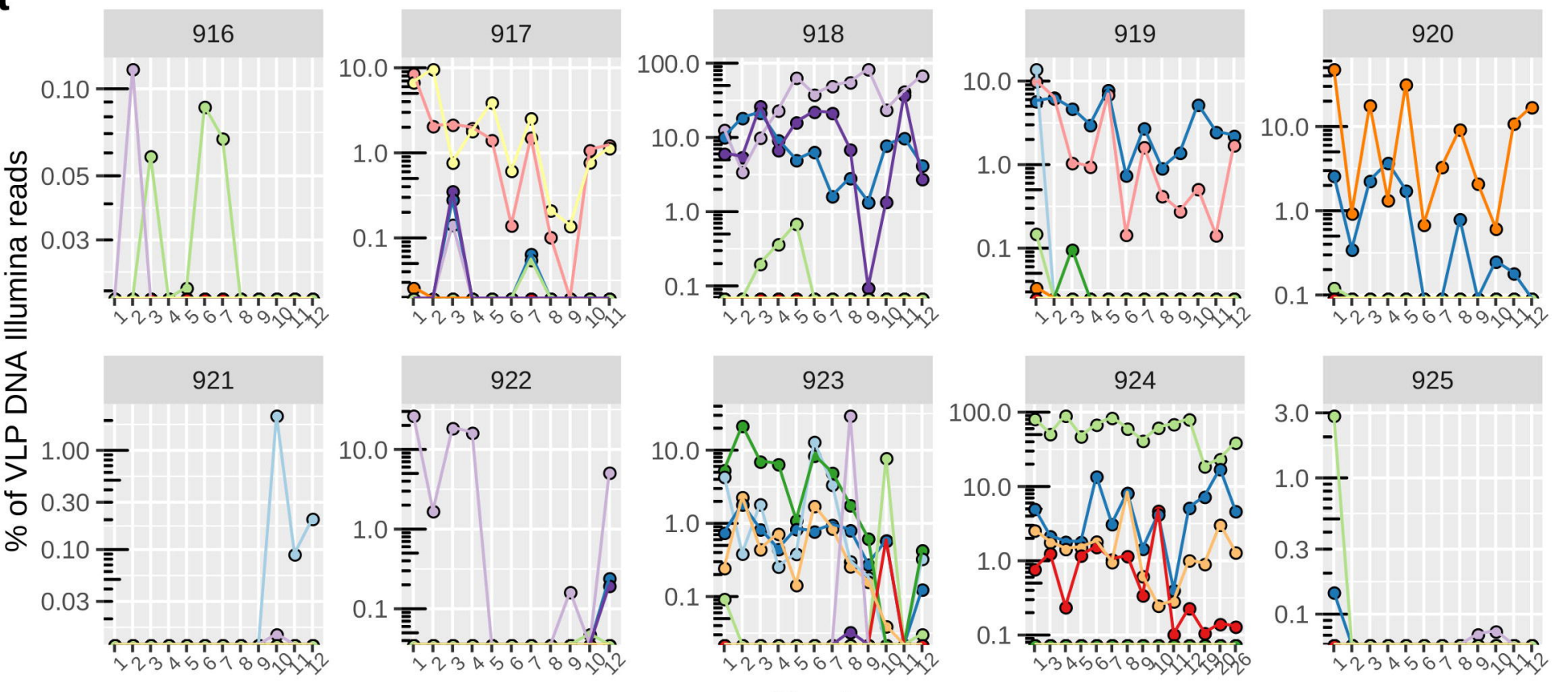

Months

b
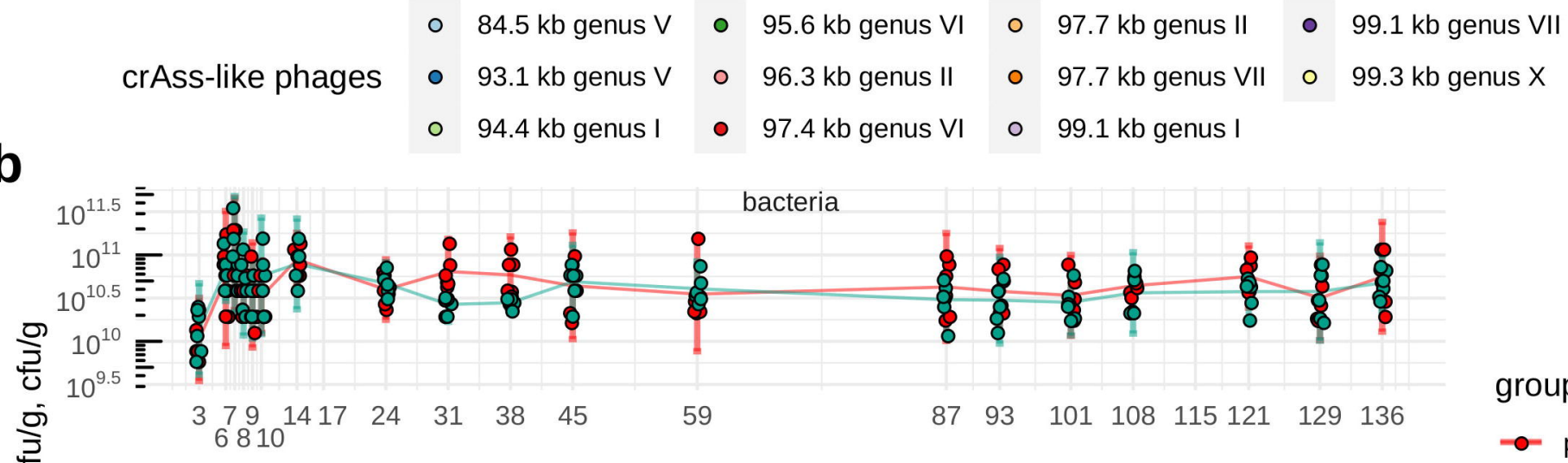

group

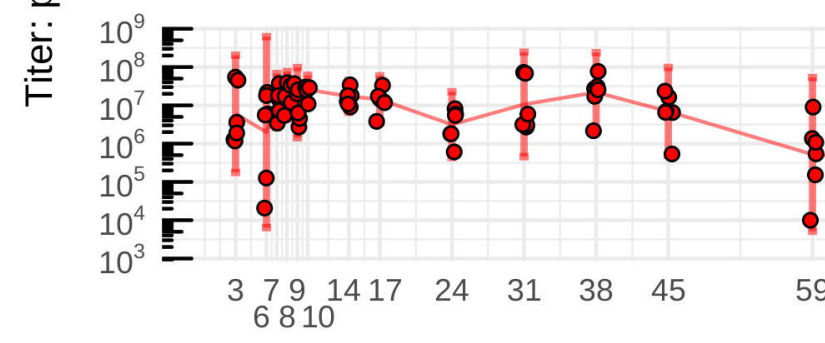

phage
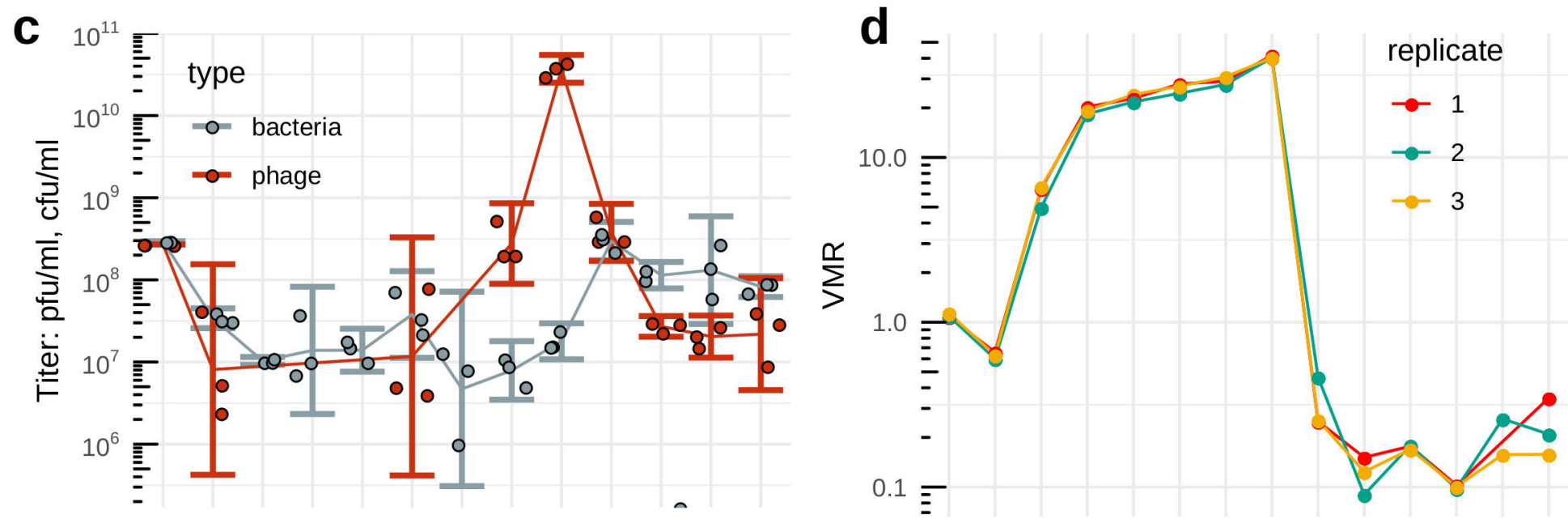

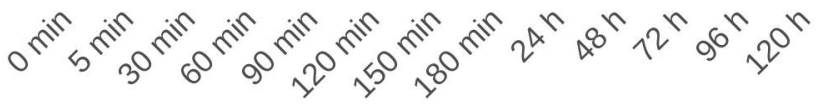


bioRxiv preprint doi: https://doi.org/10.1101/2020.12.02.408625; this version posted February 7, 2021. The copyright holder for this preprint (which was not certified by peer review) is the author/funder, who has granted bioRxiv a license to display the preprint in
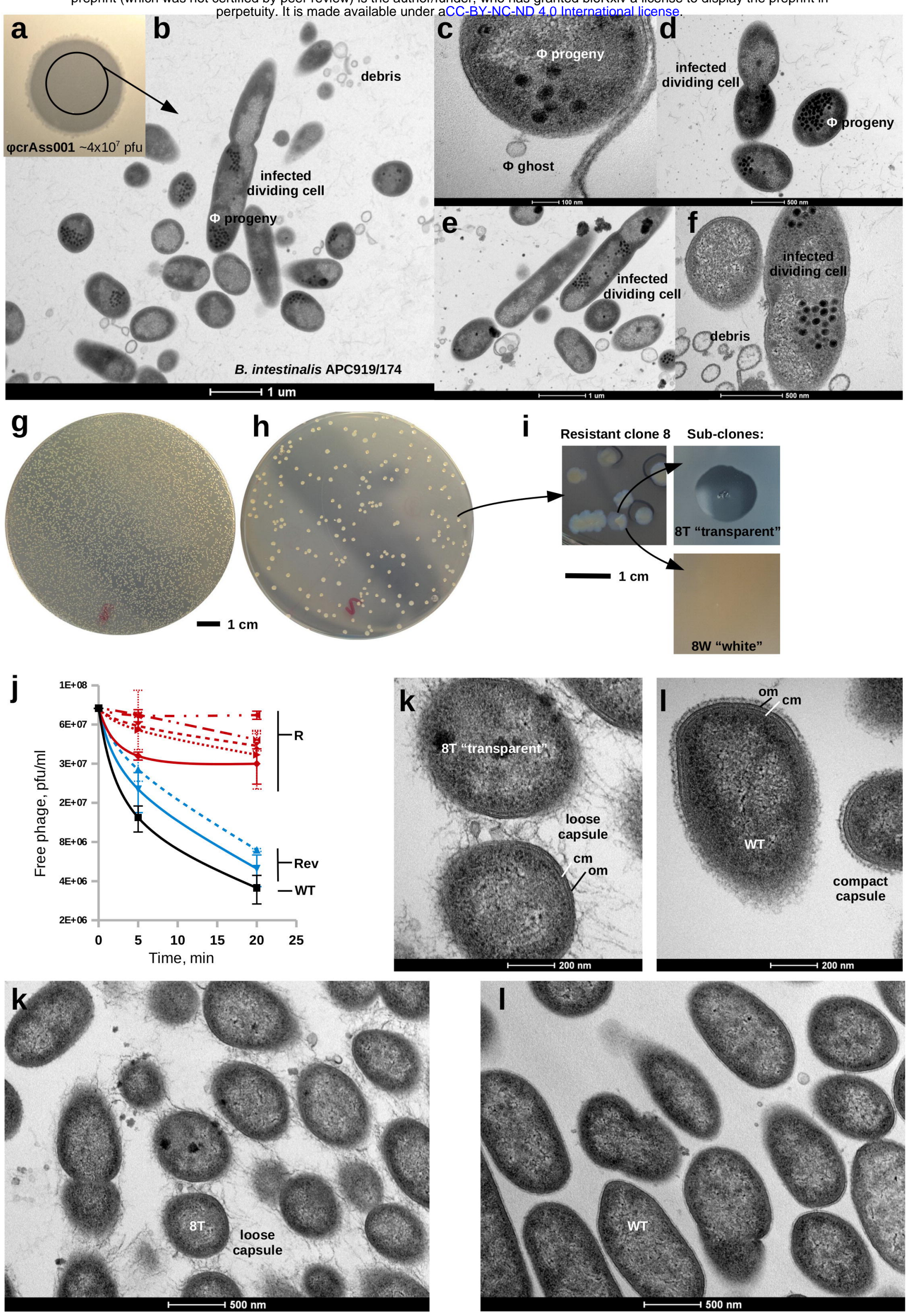


\section{$\mathbf{a}$}
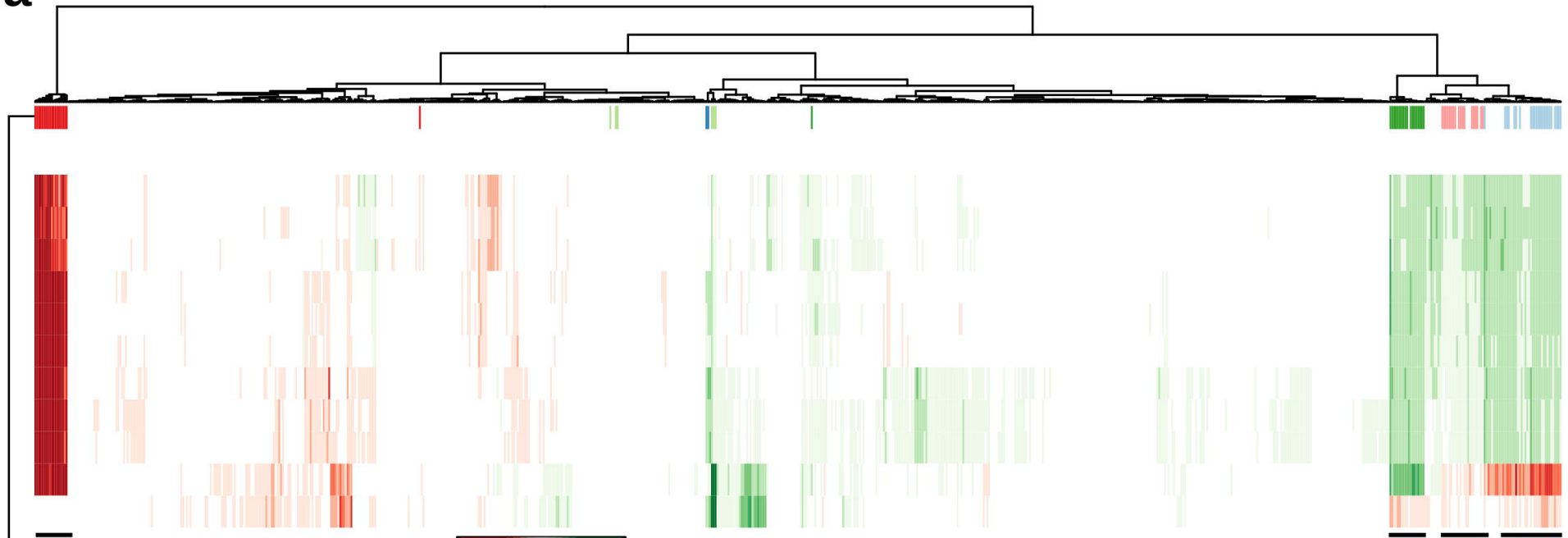

WT

Persist-24h-1

Persist-24h-2

Persist-24h-3

Persist-72h-1

Persist-72h-2

Persist-72h-3

Persist-120h-1

Persist-120h-2

Persist-120h-3

Clone-8T

$\overline{\text { PVR7 }} \overline{\text { PVR8 }} \overline{\text { PVR11 }}$

Clone-8W

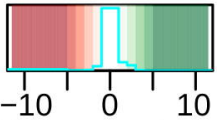

Log2 fold expression change

PVR11 PVR7

PVR3

b PVR7

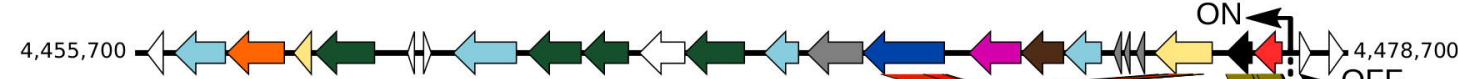

PVR8

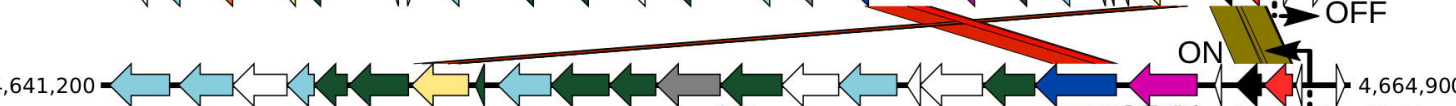

ON

$4,664,900$
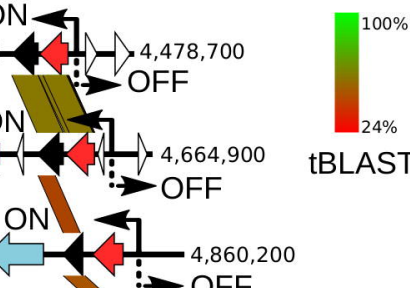

tBLASTx sequence similarity

PVR

$4,835,300 k$

PVR11

Antiterminator
$\square$ Transcriptional regulator $\square$ Dehydratase

C

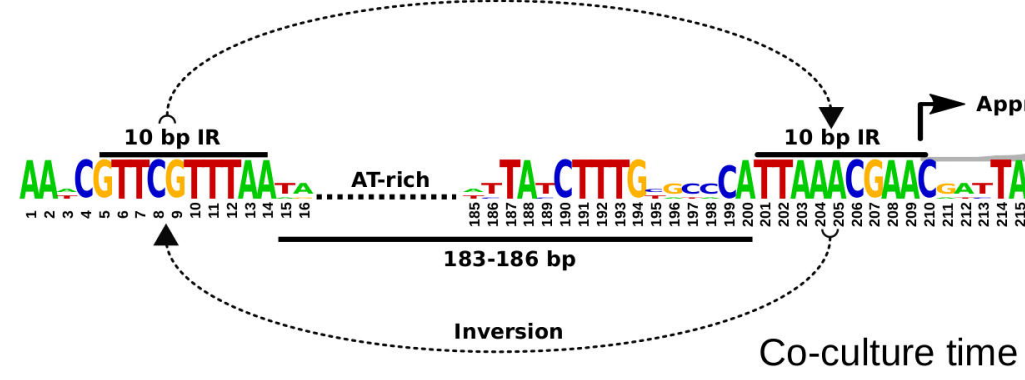

d

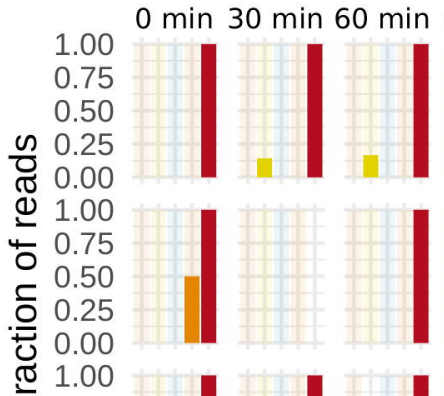

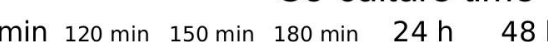
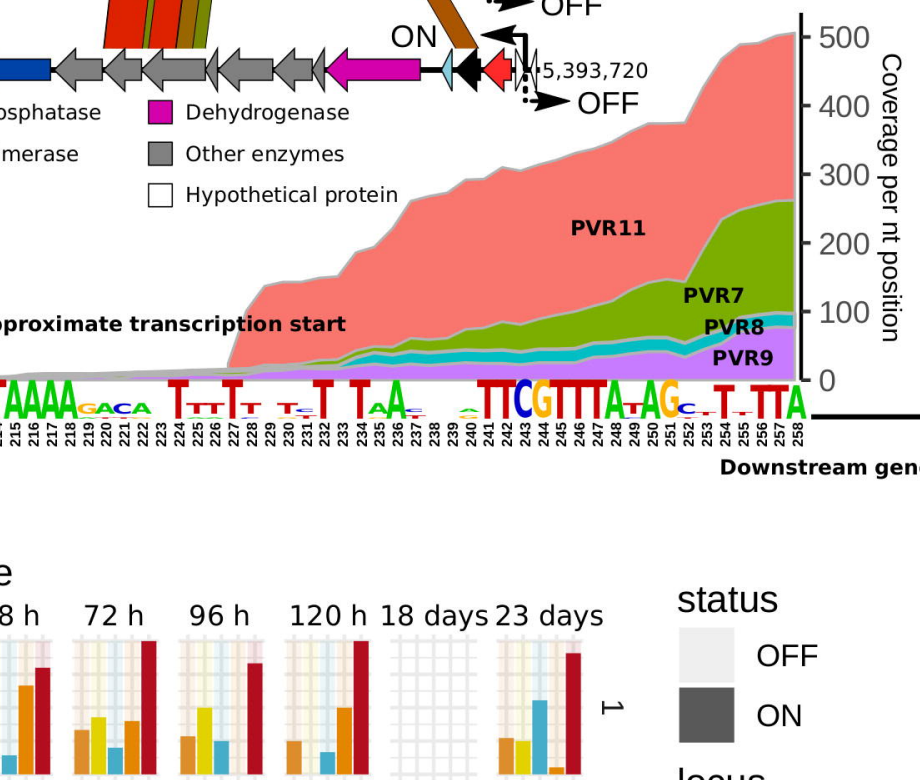

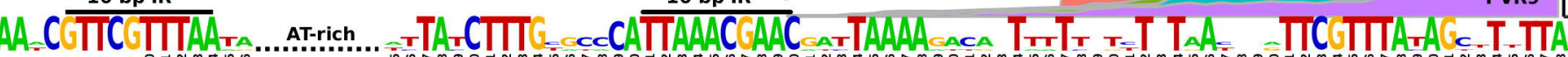

4

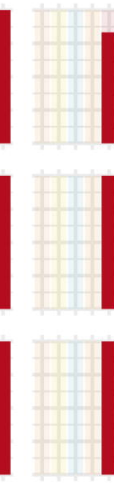

$8 \mathrm{~T}$

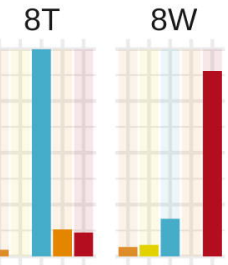

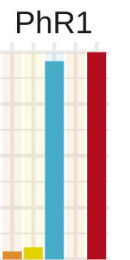

$\Perp$
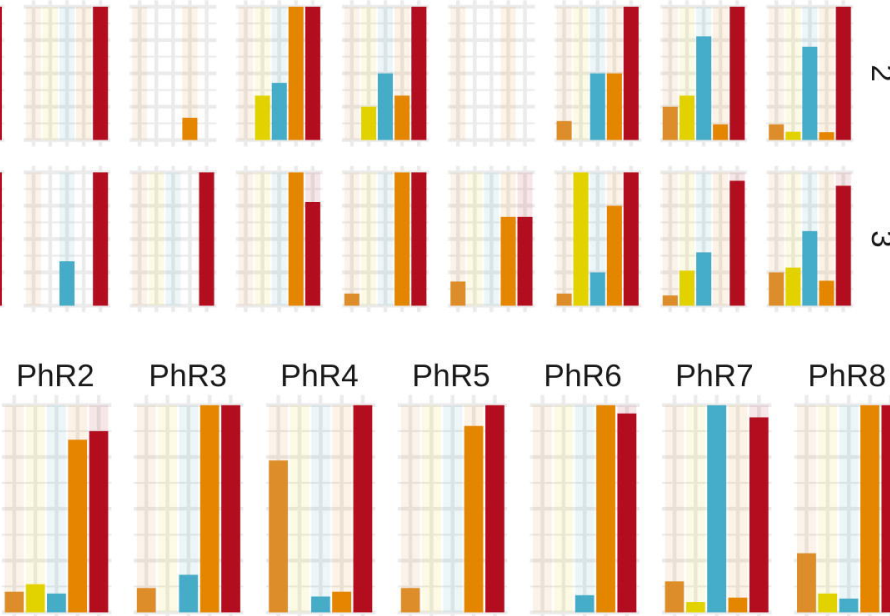

locus

PVR11

PVR12

PVR7

PVR8

PVR9 
$30 \mathrm{~min}$

25000

15000

5000

b

\section{$90 \mathrm{~min}$}

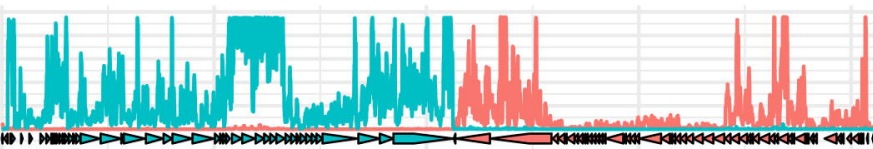

0

25000

50000

75000

100000

Coordinate in crAss001 genome

Log2 fold expression change
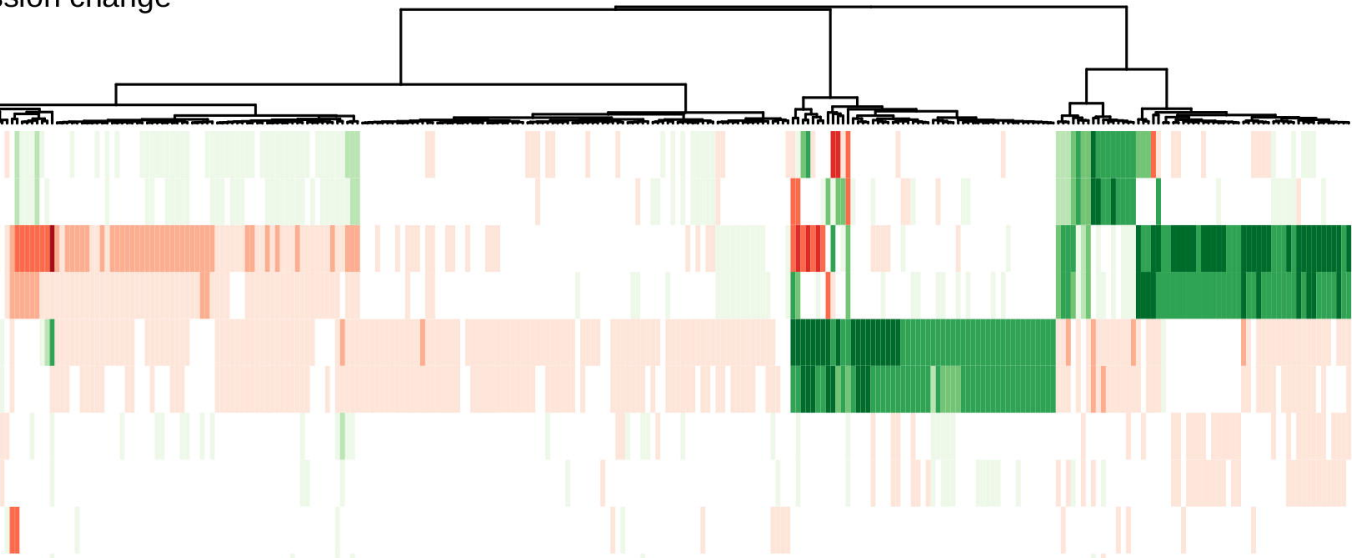

C

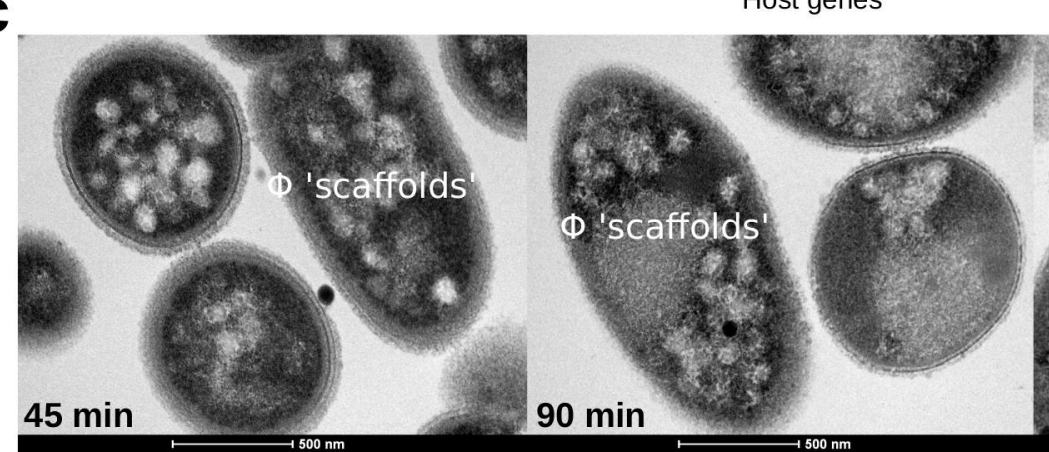

$\underset{\text { Early }}{\text { Middle }}$

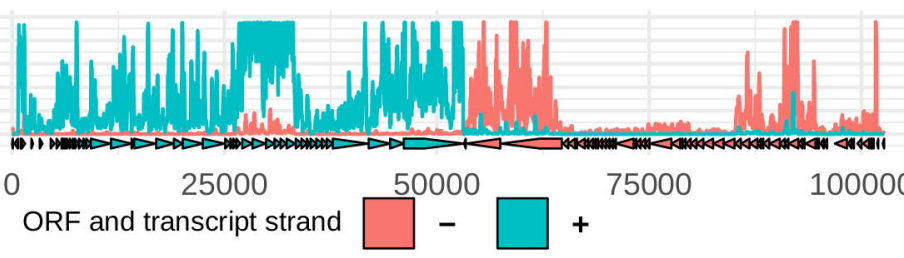

$60 \mathrm{~min}$

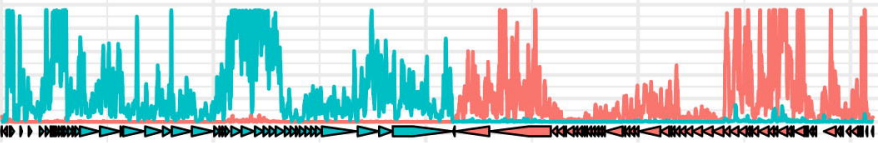

$120 \mathrm{~min}$

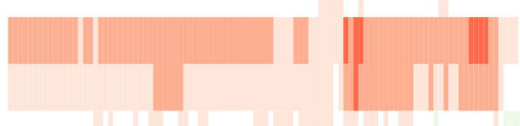

d

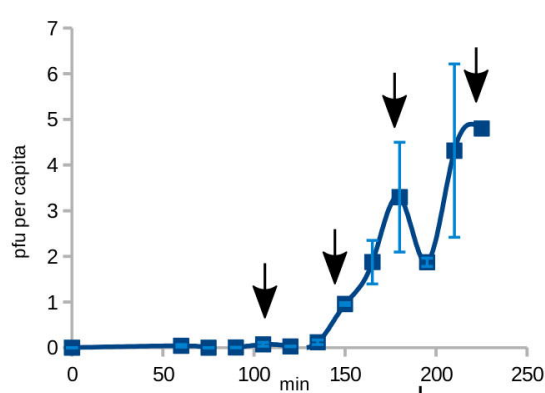

e

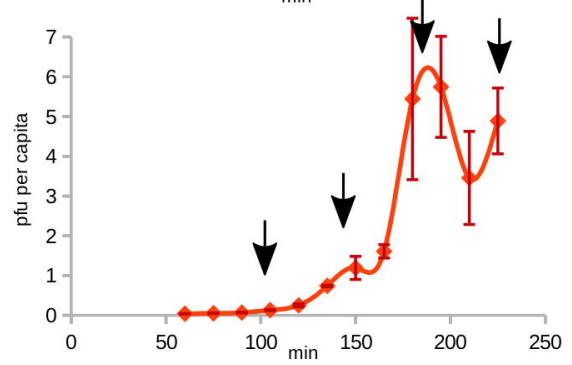

f
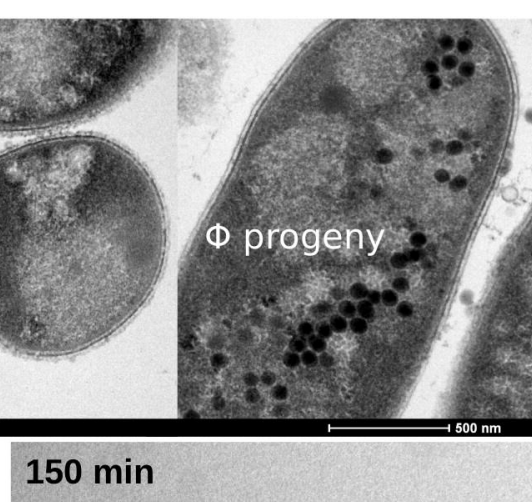

10minrun1

10minrun2

30minrun1

30minrun2

60minrun1

60minrun2

90minrun1

90minrun2

120 minrun1

120minrun2
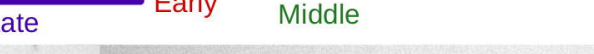
Resistant subpopulation

Phase variation subpopulation
Sensitive
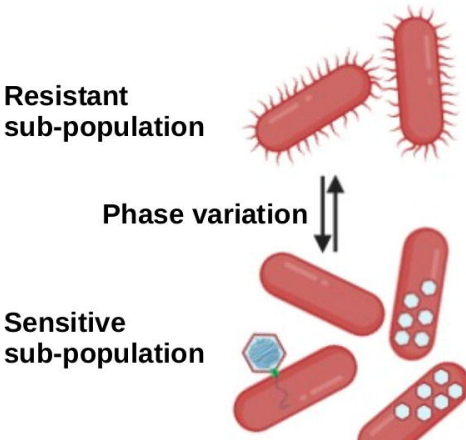